\title{
OPERATOR SPLITTING FOR AN IMMUNOLOGY MODEL USING REACTION-DIFFUSION EQUATIONS WITH STOCHASTIC SOURCE TERMS*
}

\author{
TIMOTHY A. LUCAS
}

\begin{abstract}
When immune cells detect foreign molecules, they secrete soluble factors that attract other immune cells to the site of the infection. In this paper, I study numerical solutions to a model of this behavior proposed by Kepler. In this model the soluble factors are governed by a system of reaction-diffusion equations with sources that are centered on the cells. The motion of the model cells is a Langevin process that is biased toward the gradient of the soluble factors. I have shown that the solution to this system exists for all time and remains positive, the supremum is a priori bounded and the derivatives are bounded for finite time. I have also developed a first order split scheme for solving the reaction-diffusion stochastic system. This allows us to make use of known first order schemes for solving the diffusion, the reaction and the stochastic differential equations separately.
\end{abstract}

Key words. operator splitting, reaction-diffusion, stochastic differential equations

AMS subject classifications.

1. Introduction. The immune system in higher organisms is composed of individual cells called leukocytes which work together to combat pathogens such as bacteria and viruses. These cells are unique because they move throughout the body and signal to each other in order to organize a response to an infection. Upon detecting pathogens in the body, an immune cell will secrete cytokines which are proteins that mediate communication among leukocytes and coordinate their response. Some cytokines called chemokines direct the motion of immune cells along their concentration gradient by a process known as chemotaxis. Other cytokines such as tumor necrosis factor (TNF) recruit cells to the site of inflammation and activate immune cells. One process by which inflamation is regulated is the shedding of cytokine receptors, such as those for TNF (sTNFr). Those soluble receptors bind to the respective cytokines and limit their activity. Based on these signals, the cells will group together to respond to an infection. The ability of these cells to organize spatially in a timely manner is vital to an effective immune response.

The following sections describe a mathematical model of soluble factors and immune cell motion proposed in [4] and [5]. The cytokines are modeled continuously as soluble factors governed by reaction-diffusion equations with source terms that are centered on activated leukocytes. The motion of the immune cells is inherently stochastic, but is biased toward the chemical gradients of the chemokines. We will show that solutions to this system exist and describe a numerical simulation of the model based on operator splitting. Although operator splitting is a well-known technique for solving reaction-diffusion system, there is an added degree of difficulty due to the stochastic source terms.

1.1. Soluble Factors. Suppose $U_{i}(x, t)$ is the local concentration of soluble factor $i$ at the position $x$ and time $t$. Let $x_{\mu}$ be the position of the center of cell $\mu$ in the bounded domain $\Omega \in \mathbb{R}^{3}$. Then for $x \in \Omega$, the reaction-diffusion equations that

${ }^{*}$ This work was supported by the National Institute of Health through grants 1R21-A1058227-02 and HHSN266200500019C. 
describe the behavior of the soluble factors from [5] are

$$
\frac{\partial U_{i}}{\partial t}(x, t)=\left\{D_{i} \Delta-\sum_{j=1}^{N} r_{i j} U_{j}(x, t)-\lambda_{i}\right\} U_{i}(x, t)+\sum_{\mu=1}^{M} J_{i \mu}(x, t) g_{\mu}\left(x-x_{\mu}(t)\right)
$$

where $g_{\mu}(x)$ is a smoothly cut-off Gaussian with support $\{x:|x|<\mu\}$. Here $D_{i}$ denotes the diffusion coefficient for soluble factor $U_{i}, r_{i j}$ is the rate at which $U_{i}$ is removed by interaction with $U_{j}$, and $\lambda_{i}$ is the rate of removal of $U_{i}$ by other factors. The secretion of soluble factor $U_{i}$ by cell $\mu$ is a Gaussian source centered at the cell position $x_{\mu}$ with secretion rate $J_{i \mu}$. The source term in (1.1) is a sum over the $M$ individual cells indexed by $\mu$. The constants $D_{i}, r_{i j}$, and $J_{i \mu}$ are all positive.

In this paper we consider a smooth source term with compact support as opposed to the point sources in [5] for two reasons. Point sources do not take into account the volume of the cells which can vary in size and shape. Also, the point sources in [5] are delta functions and we are currently unaware of any rigorous mathematical treatment of (1.1) with this type of source. We will make use of the smoothness of the source terms in order to show the existence of a solution.

1.2. Cell Motion. The system (1.1) is coupled with $M$ systems of stochastic differential equations that describe the motion of each individual cell. For each cell, indexed by $\mu$, we let $x_{t} \in \mathbb{R}^{3}$ be the position and $v_{t} \in \mathbb{R}$ be the velocity The motion of each cell is a Langevin process

$$
\begin{aligned}
d x_{t} & =v_{t} d t \\
d v_{t} & =\left[h\left(U\left(x_{t}\right)\right)-\gamma v\right] d t+\sigma \sqrt{\gamma} d W_{t}
\end{aligned}
$$

where $d W_{t}$ is standard Brownian motion in $\mathbb{R}^{3}$. The direction of motion is random, but biased toward the direction of the vector $h(U)$ which depends on the local concentration of one or more of the soluble factors by the relation

$$
h(U)=\sum_{i=1}^{N} \frac{\chi_{i} \nabla U_{i}}{h_{0}+\left|\nabla U_{i}\right|} .
$$

Here $\chi_{i}$ is the chemotactic constant that controls how much the drift is influenced by the gradient of soluble factor $U_{i}$ and $h_{0}$ is the length of the gradient of $U_{i}$ at which $h$ attains half of its maximum value. The constants $\gamma, h_{0}$ and $\chi_{i}$ are assumed to be positive.

1.3. Notation. Throughout we will refer to $C_{f}\left(x_{1}, x_{2}, \ldots, x_{n}\right)$ as a constant that depends on the function $f$ and the values of $x_{1}, x_{2}, \ldots, x_{n}$. We also refer to $L_{f}\left(x_{1}, x_{2}, \ldots, x_{n}\right)$ as a Lipschitz constant for the function $f$ that depends on $x_{1}, x_{2}, \ldots, x_{n}$. We define $B_{r}(x)$ to be a ball of radius $r$ centered at $x \in \mathbb{R}^{n}$ and $\mathrm{Cu}(a, b, n)$ to be the hypercube in $\mathbb{R}^{n}$ that extends from $a$ to $b$ in every direction. For $a, b \in \mathbb{R}$ we define $a \vee b=\max (a, b)$ and $a \wedge b=\min (a, b)$.

Let $X$ and $Y$ be sets and suppose that $f_{i}: X \rightarrow Y, i=1, \ldots, n$. Then $\left(f_{1}, \ldots, f_{n}\right)$ is an $n$-tuple whose components are in $Y^{X}$. We shall also let $\left(f_{1}, \ldots, f_{n}\right)$ denote the function from $X$ to $Y^{n}$ which assigns $\left(f_{1}(x), f_{2}(x), \ldots f_{n}(x)\right)$ to $x \in X$, i.e.

$$
\left(f_{1}, f_{2}, \ldots, f_{n}\right)(x)=\left(f_{1}(x), f_{2}(x), \ldots f_{n}(x)\right) .
$$


1.4. Overview and Main Results. In the following sections we consider the case where $\Omega$ is a cube and the reaction-diffusion equations for the soluble factors have periodic boundary conditions. Section 2 contains a proof that the reactiondiffusion-stochastic system (1.1), (1.2) has a solution for a short time. We consider a first order split scheme in $\S 3$ in order to make use of well known numerical schemes for solving the diffusion, reaction, and stochastic differential equations for the cell motion. In section $\S 4$, we carefully add up the local truncation error due to the splitting using a method similar to the convergence proof of higher order numerical schemes for stochastic differential equations by Milstein in [9]. We also find in $\S 4$ that the solution to the reaction-diffusion system with a nonnegative initial condition is positive, the supremum is a priori bounded and that derivatives are bounded for finite time. In addition, solutions to the reaction-diffusion-stochastic system exist for all time.

\section{Short Time Existence.}

2.1. Preliminaries. Suppose the domain $\Omega$ is an open cube in $\mathbb{R}^{n}$ with side length $\Lambda$. Let $\mathbb{Z}^{n}$ be the set of integer frequencies $\omega=\left(\omega_{1}, \ldots, \omega_{n}\right)$ and define $|\omega|_{\infty}=$ $\max \left|\omega_{i}\right|$. Let $A(k, n)$ be the set of multi-indices $\alpha=\left(\alpha_{1}, \alpha_{2}, \ldots \alpha_{n}\right)$ such that

$$
w(\alpha)=\sum_{i=1}^{n}\left|\alpha_{i}\right| \leq k .
$$

Let $\mathcal{E}$ be the vector space of smooth $\Lambda$-periodic complex valued functions on $\mathbb{R}^{n}$. For each $\omega \in \mathbb{Z}^{n}$ define $E_{\omega}(x) \in \mathcal{E}$ by

$$
E_{\omega}(x)=\Lambda^{-\frac{n}{2}} e^{\frac{i 2 \omega \cdot x}{\Lambda}}, \quad x \in \Omega .
$$

For a locally integrable function $f$ define the Fourier coefficient

$$
c_{\omega}(f)=\int_{\Omega} f(x) E_{\omega}(x) d x .
$$

For $s \in \mathbb{R}$ and a locally integrable function $f$, let $\|f\|_{s} \in[0, \infty]$ be such that

$$
\|f\|_{s}^{2}=\sum_{\omega \in \mathbb{Z}^{n}}\left(1+|\omega|_{\infty}^{2}\right)^{s}\left|c_{\omega}(f)\right|^{2} .
$$

Definition 2.1. For $s \geq 0$, define $\mathbb{H}_{s}$ to be the space of measurable, $\Lambda$-periodic, complex valued functions $f$ on $\Omega \subset \mathbb{R}^{n}$ such that $\|f\|_{s}<\infty$.

REMARK 2.2. If $k>\frac{n}{2}$, then corresponding to any element $f \in \mathbb{H}_{k}$ is a continuous function $g \in \mathbb{H}_{k}$ such that $f=g$ almost everywhere. We may therefore assume

when $k>\frac{n}{2}$ that the elements of $\mathbb{H}_{k}$ are continuous. It is well known that the set $\left\{f \in \mathbb{H}_{k}: f\right.$ is continuous $\}$ is a Hilbert space with respect to $\|\cdot\|_{k}$.

A proof of the following proposition is given in [2].

Proposition 2.3. Suppose $f \in \mathbb{H}_{k}$. For any $\alpha \leq k$, there exists $g_{\alpha} \in \mathbb{H}_{k-w(\alpha)}$ such that

$$
\int_{\Omega} f(x) \partial^{\alpha} \phi(x) d x=(-1)^{w(\alpha)} \int_{\Omega} g_{\alpha} \phi(x) d x, \quad \phi \in \mathcal{E} .
$$

Let $g_{\alpha}$ satisfy the conditions above. Then

$$
\|f\|_{k}^{2}=\sum_{w(\alpha) \leq k}\left\|g_{\alpha}\right\|_{0}^{2} .
$$


The following lemma is useful throughout and the proof is also found in [2].

Lemma 2.4 (Sobolev's Lemma). Let $k>\frac{n}{2}+l$. Then

$$
\sup _{w(\alpha) \leq l}\left|\partial^{\alpha} f(x)\right| \leq C(k, l)\|f\|_{k} .
$$

REMARK 2.5. Note that $f \in \mathbb{H}_{k+l}$ if and only if $\partial^{\alpha} f \in \mathbb{H}_{k}$ for $w(\alpha) \leq l$.

Define the linear map $\Delta: \mathbb{H}_{4} \rightarrow \mathbb{H}_{2}$ by

$$
\Delta f=\sum_{i=1}^{3}\left(\frac{\partial}{\partial x_{i}}\right)^{2} f
$$

Then for $s \geq 4,\|(1-\Delta)(f)\|_{s}=\|f\|_{s+2}$, since

$$
(1-\Delta) f=\sum_{\omega \in \mathbb{Z}^{n}} c_{\omega}(f)\left(1-\frac{\partial^{2}}{\partial x_{i}{ }^{2}}\right) E_{\omega}(x)=\sum_{\omega \in \mathbb{Z}^{n}} c_{\omega}(f)\left(1+|\omega|^{2}\right) E_{\omega}(x) .
$$

Let $\mathbb{H}_{k}^{N}$ be the space of $N$-tuples $u=\left(u_{1}, u_{2}, \ldots, u_{N}\right)$ such that $u_{i} \in \mathbb{H}_{k}$ for $i=1,2 \ldots, N$ and set

$$
\|u\|_{k}=\left(\sum_{i=1}^{N}\left\|u_{i}\right\|_{k}^{2}\right)^{\frac{1}{2}}
$$

By our convention $\left(u_{1}, \ldots, u_{N}\right)(x)=\left(u_{1}(x), \ldots, u_{N}(x)\right)$. Define $\mathcal{D}: \mathbb{H}_{4}^{N} \rightarrow \mathbb{H}_{2}^{N}$ by

$$
\mathcal{D}(u)=\left(D_{1} \Delta u_{1}, D_{2} \Delta u_{2}, \ldots, D_{N} \Delta u_{N}\right)
$$

where $D_{i}>0$ for $i=1,2 \ldots, N$. We now define the fundamental solution of the diffusion equation. For each $t>0$, define the linear map $K_{t, i}: \mathbb{H}_{k} \rightarrow \mathcal{E}$ by

$$
K_{t, i}\left(u_{i}\right)=\sum_{\omega \in \mathbb{Z}^{n}} e^{-\frac{2 D_{i}|w|^{2} t}{\Lambda}} c_{\alpha}\left(u_{i}\right) E_{\omega}
$$

Then

$$
\frac{d}{d t} K_{t, i}\left(u_{i}\right)=D_{i} \Delta K_{t, i}\left(u_{i}\right)=K_{t, i}\left(D_{i} \Delta u_{i}\right)
$$

For $t>0$ define $K_{t}: \mathbb{H}_{k}^{N} \rightarrow \mathcal{E}^{N}$ by

$$
K_{t}(u)=\left(K_{t, 1}\left(u_{1}\right), K_{t, 2}\left(u_{2}\right), \ldots, K_{t, N}\left(u_{N}\right)\right)
$$

and note that

$$
\frac{d}{d t} K_{t}(u)=\mathcal{D}\left(K_{t}(u)\right)=K_{t}(\mathcal{D}(u))
$$

It also follows that

$$
\left\|K_{t} u_{i}\right\|_{s} \leq\left\|u_{i}\right\|_{s}, \quad i=1,2, \ldots, N, \quad \text { and } \quad\left\|K_{t} u\right\|_{s} \leq\|u\|_{s} .
$$

Below we define the space of solutions to the reaction-diffusion equations (1.1). 
Definition 2.6. For any integer $k>\frac{n}{2}$, let $\mathbf{B}_{k}^{N}\left(m_{1}, m_{2}\right)$ be the set of $N$-tuples $u \in \mathbb{H}_{k}^{N}$ such that $\sup |u| \leq m_{1}$ and $\|u\|_{k} \leq m_{2}$. Note that

$$
\mathbb{H}_{k}^{N}=\bigcup_{0 \leq m_{1}, m_{2}<\infty} \mathbf{B}_{k}^{N}\left(m_{1}, m_{2}\right) .
$$

REMARK 2.7. If $u \in \mathbf{B}_{k}^{N}\left(m_{1}, m_{2}\right)$ and $t>0$, then $\sup \left|K_{t}(u)\right| \leq \sup |u|$.

DEFINITION 2.8. For any integer $k>\frac{n}{2}$, let $\mathbf{C}_{k, T}^{N}\left(m_{1}, m_{2}\right)$ be the set of functions $U:[0, T] \rightarrow \mathbf{B}_{k}^{N}\left(m_{1}, m_{2}\right)$ that are continuous with respect to $\|\cdot\|_{k}$. Then define

$$
\|U\|_{k, T}=\sup _{0 \leq t \leq T}\|U(t)\|_{k} .
$$

Also, let

$$
\mathbf{C}_{k, T}^{N}=\bigcup_{0 \leq m_{1}, m_{2}<\infty} \mathbf{C}_{k, T}^{N}\left(m_{1}, m_{2}\right) .
$$

Below we define the space of solutions to the stochastic differential equations (1.2).

Definition 2.9. Suppose $T>0$ and $m>0$. Define $\mathcal{S}_{T}(m)$ to be the space of continuous stochastic processes such that the norm

$$
\left\|X_{t}\right\|_{\mathcal{S}_{T}}=\left(\mathbb{E}\left[\sup _{0 \leq t \leq T}\left|X_{t}\right|^{2}\right]\right)^{\frac{1}{2}} \leq m .
$$

We now define the space of functions which will contain the solutions to the reaction-diffusion-stochastic system (1.1),(1.2).

DeFINITION 2.10. For any integer $k>\frac{n}{2}$, let $\mathcal{B}_{k}^{N}\left(m_{1}, m_{2}, m_{3}\right)$ be the space of ordered pairs $\phi=(u, \xi)$ where $u \in \mathbf{B}_{k}^{N}\left(m_{1}, m_{2}\right)$ and $\xi$ is a random variable such that $\mathbb{E}\left[|\xi|^{2}\right]^{\frac{1}{2}} \leq m_{3}$. Then define

$$
\|\phi\|_{k}=\mathbb{E}\left[\|u\|_{k}^{2}+|\xi|^{2}\right]^{\frac{1}{2}}
$$

Definition 2.11. For any integer $k>\frac{n}{2}$ and $T>0$, let $\mathcal{C}_{k, T}^{N}\left(M_{1}, M_{2}, M_{3}\right)$ be the Cartesian product of $\mathbf{C}_{k, T}^{N}\left(M_{1}, M_{2}\right)$ and $\mathcal{S}_{T}\left(M_{3}\right)$. Define the norm $\|\cdot\|_{k, T}$ by

$$
\|(U, X)\|_{k, T}=\mathbb{E}\left[\sup _{0 \leq t \leq T}\left(\|U(t)\|_{k}^{2}+\left|X_{t}\right|^{2}\right)\right]^{\frac{1}{2}}
$$

Since our space of solutions is periodic, we must define the Gaussian source terms to be periodic as well. Let $g: \mathbb{R}^{n} \rightarrow \mathbb{R}$ be a mean zero Gaussian with standard deviation $\mu$. Define $g_{\mu, i}$ to be the periodification of $g_{\mu}$, i.e.

$$
g_{\mu, i}(x)=\sum_{j \in \mathbb{Z}} g_{\mu}\left(x-\frac{2 \pi j}{\Lambda}\right) .
$$

Let $\bar{J}_{i}>0$ for $i=1,2, \ldots, N$ and $J=\max \left\{\bar{J}_{i}: i=1,2, \ldots, N\right\}$. Then let $\epsilon>0$ and $J_{i}: \mathbb{R}^{n} \rightarrow \mathbb{R}$ be a smooth cutoff defined by

$$
J(X)=\left\{\begin{array}{cc}
\bar{J}_{i} & X \in \mathrm{Cu}\left(-\frac{\Lambda}{2}+\epsilon, \frac{\Lambda}{2}-\epsilon\right) \\
0 & X \notin \mathrm{Cu}\left(-\frac{\Lambda}{2}, \frac{\Lambda}{2}\right)
\end{array}\right.
$$


Then define $g: \mathbb{R}^{n} \times \mathbb{R}^{n} \rightarrow \mathbb{R}^{N}$ by

$$
g(X, x)=\left(J_{1}(X) g_{1}(x), \ldots, J_{N}(X) g_{N}(x)\right) .
$$

Let $T>0,(U, X) \in \mathcal{C}_{k, T}^{N}$ and $\mathcal{T}_{x}$ be translation by $x \in \mathbb{R}^{n}$. Throughout we will consider the reaction-diffusion-stochastic system with one cell,

$$
\begin{aligned}
\frac{d U}{d t} & =\mathcal{D} U(t)+f(U)(t)+g\left(X_{t}\right) \circ \mathcal{T}_{X_{t}}, \quad 0<t<T, \\
d X_{t} & =a\left(U\left(X_{t}\right), X_{t}\right) d t+b\left(X_{t}\right) d W_{t}, \quad 0<t<T, \\
(U, X)(0) & =(u, \xi)
\end{aligned}
$$

where $u \in \mathbb{H}_{k}^{N}$ and $\xi \in \mathbb{R}^{n}$. We will usually consider the case where $n=3$.

REMARK 2.12. We compose $g$ with $\mathcal{T}_{X_{t}}$ so that the Gaussian source term is centered on the position of the cell. If the cell leaves the domain, then the cutoff $J$ restricts the source term $g$ from contributing to the reaction-diffusion equation. Since $J_{i}$ is a function of $X, g$ is still periodic in $x$.

2.2. Estimates for the Reaction-Diffusion Equations. The following theorem provides estimates for the reaction terms $f(u)$.

THEOREM 2.13. Let $N$ be a positive integer and $f: \mathbb{R}^{N} \rightarrow \mathbb{R}^{N}$ be a smooth function such that $f(0)=0$. Suppose $0 \leq m_{1}, m_{2}<\infty, k$ is a nonnegative integer and $u, v \in \mathbf{B}_{k}^{N}\left(m_{1}, m_{2}\right)$. Then

$$
\begin{gathered}
|f(u)| \leq c_{f, 1}\left(m_{1}\right)|u|, \\
\|f(u)\|_{k} \leq C_{f, k}\left(m_{1}\right)\|u\|_{k},
\end{gathered}
$$

where

$$
\begin{aligned}
& c_{f, k}\left(m_{1}\right)=\sup \left\{\left|\left(\frac{\partial}{\partial u}\right)^{\alpha} f(u)\right|: w(\alpha) \leq k, u \in \mathbb{R}^{N} \text { and }|u| \leq m_{1}\right\}, \\
& C_{f, k}\left(m_{1}\right)=C(k, N) c_{f, \max \{1, k\}}\left(m_{1}\right)\left(1+m_{1}\right)^{\max \{0, k-1\})} .
\end{aligned}
$$

Let $k=0$ or $k>\frac{n}{2}$. Then we also have

$$
\|f(u)-f(v)\|_{k} \leq L_{f, k}\left(m_{1}, m_{2}\right)\|u-v\|_{k}
$$

where

$$
\begin{aligned}
L_{f, 0}\left(m_{1}\right) & =c_{f, 1}\left(m_{1}\right), \\
L_{f, k}\left(m_{1}, m_{2}\right) & =C(k, N) c_{f, k+1}\left(m_{1}\right)\left(1+m_{1}\right)^{k-1} m_{2} .
\end{aligned}
$$

Proof. By the Fundamental Theorem of Calculus,

$$
\begin{aligned}
f(u)-f(v) & =\int_{0}^{1} \frac{d}{d t} f((1-t) v+t u) d t \\
& =\left(\int_{0}^{1} \partial f((1-t) v+t u) \frac{d}{d t}((1-t) v+t u) d t\right) \\
& =\left(\int_{0}^{1} \partial f((1-t) v+t u) d t\right)(u-v) .
\end{aligned}
$$


It follows by the Cauchy-Schwarz inequality that

$$
\|f(u)-f(v)\|_{0} \leq c_{f, 1}\left(m_{1}\right)\|u-v\|_{0}
$$

and for $v=0$,

$$
|f(u)| \leq c_{f, 1}\left(m_{1}\right)|u| \quad \text { and } \quad\|f(u)\|_{0} \leq c_{f, 1}\left(m_{1}\right)\|u\|_{0} .
$$

Let $k$ be a positive integer. Using well known arguments found in [11], 113.3 , there exist constants $C(k, N)$ and $C(k)$ such that

$$
\|f(u)\|_{k} \leq C(k, N) c_{f, k}\left(m_{1}\right)\left(1+m_{1}\right)^{k-1}\|u\|_{k},
$$

and

$$
\left\|u_{1} u_{2}\right\|_{k} \leq C(k)\left(\left\|u_{1}\right\|_{k}\left\|u_{2}\right\|_{\infty}+\left\|u_{1}\right\|_{\infty}\left\|u_{2}\right\|_{k}\right)
$$

Then (2.21) immediately follows from (2.26). Let $u_{1}=\int_{0}^{1} \partial f((1-t) v+t u) d t$ and $u_{2}=u-v$. Then by $(2.26)$,

$$
\left\|u_{1}\right\|_{k} \leq C(k+1, N) c_{f, k+1}\left(m_{1}\right)\left(1+m_{1}\right)^{k}\left(\|u\|_{k} \vee\|v\|_{k}\right) .
$$

We also have $\left\|u_{1}\right\|_{\infty} \leq c_{f, 1}\left(m_{1}\right)$. The Sobolev Lemma gives us

$$
\|u-v\|_{\infty} \leq C(k)\|u-v\|_{k} .
$$

Finally, by (2.27)

$$
\begin{aligned}
\|f(u)-f(v)\|_{k} & =\left\|\left(\int_{0}^{1} \partial f((1-t) v+t u) d t\right)(u-v)\right\|_{k} \\
& \leq C(k+1, N) c_{f, k+1}\left(m_{1}\right)\left(1+m_{1}\right)^{k}\left(\|u\|_{k} \vee\|v\|_{k}\right)\|u-v\|_{k}
\end{aligned}
$$

which proves $(2.24)$.

REMARK 2.14. If $k>\frac{n}{2}$, then we can apply the Sobolev Lemma to equations (2.21) and (2.24) The resulting bounds are

$$
\begin{aligned}
\|f(u)\|_{k} & \leq C_{f, k}\left(m_{2}\right), \\
\|f(u)-f(v)\|_{k} & \leq L_{f, k}\left(m_{2}\right)\|u-v\|_{k} .
\end{aligned}
$$

The following estimates on the source terms $g$ can be easily obtained.

Lemma 2.15. Let $g$ be given by (2.18). Then for an integer $k \geq 0$, there exist constants $C_{g}, C_{g, k}$ and $L_{g, k}$ such that

$$
\left|g \circ \mathcal{T}_{y}\right| \leq C_{g}, \quad\left\|g \circ \mathcal{T}_{y}\right\|_{k} \leq C_{g, k} \quad \text { and } \quad\left\|g \circ \mathcal{T}_{y}-g \circ \mathcal{T}_{z}\right\|_{k} \leq L_{g, k}|y-z| .
$$

2.3. Estimates for the Cell Motion. Let $X_{t}=\left(x_{t}, v_{t}\right)$ and

$$
a\left(U, X_{t}\right)=\left(\begin{array}{c}
v_{t} \\
h(U)-\gamma v_{t}
\end{array}\right) \text { and } b\left(X_{t}\right)=\left(\begin{array}{cc}
0 & 0 \\
0 & \sigma \sqrt{\gamma}
\end{array}\right) .
$$


It is immediately clear that $b$ is Lipschitz in $X$ and bounded. Recall that $a$ depends on $U\left(X_{t}\right)$ by the relation

$$
h\left(U\left(X_{t}\right)\right)=\sum_{i=1}^{N} \frac{\chi_{i} \nabla U_{i}\left(x_{t}\right)}{\left.1+\mid \nabla U_{i}\left(x_{t}\right)\right) \mid} .
$$

Since $h(U)$ is bounded by $N\left(\max _{1 \leq i \leq N} \chi_{i}\right)$,

$$
|a(u(X), X)| \leq C_{a}(m), \quad X \in \mathcal{S}_{T}(m) .
$$

Using the Sobolev Lemma we can show that $a$ is Lipschitz in both $u$ and $X$.

Lemma 2.16. The drift $a(u(X), X)$ is Lipschitz in $u$ and $X$, i.e. there exist constants $L_{a, 1}\left(M_{2}\right)$ and $L_{a, 2}\left(M_{2}\right)$ such that

$$
|a(u(X), X)-a(v(Y), Y)| \leq L_{a, 1}\left(M_{2}\right)\|u-v\|_{3}+L_{a, 2}\left(M_{2}\right)|X-Y|
$$

for $u, v \in \mathbf{B}_{4}^{N}\left(M_{1}, M_{2}\right)$.

The proof of this lemma depends on the fact that the supremum is bounded by the $\mathbb{H}_{s}$ norm for $s>\frac{3}{2}$ in three dimensions. Therefore, the supremum of the difference between $u$ and $v \in B_{4}^{N}$ is bounded by the $\mathbb{H}_{3}$ norm and a Lipschitz constant for $\nabla u$ is $\|u\|_{4}$. Also $\nabla u$ is Lipschitz in $x$ with a constant depending on $\|u\|_{4}$.

The following facts about stochastic integrals from [8] and [10] are useful throughout.

Definition 2.17. Let $\mathcal{M}^{2}\left(\left[t_{1}, t_{2}\right], \mathbb{R}^{n}\right)$ be the space of measurable, $\mathcal{F}_{t}$ adapted processes $f:\left[t_{1}, t_{2}\right] \rightarrow \mathbb{R}^{n}$ such that

$$
\mathbb{E}\left[\int_{t_{1}}^{t_{2}}|f(t, \omega)|^{2} d t\right]<\infty
$$

Proposition 2.18 (Itô Isometry). Let $f \in \mathcal{M}^{2}\left(\left[t_{1}, t_{2}\right], \mathbb{R}^{n}\right)$. Then

$$
\mathbb{E}\left[\left|\int_{t_{1}}^{t_{2}} f(t, \omega) d W_{t}\right|^{2}\right]=\mathbb{E}\left[\int_{t_{1}}^{t_{2}}|f(t, \omega)|^{2} d t\right] .
$$

Proposition 2.19 (Doob's Martingale Inequality for Stochastic Integrals). Let $f \in \mathcal{M}^{2}\left([0, T], \mathbb{R}^{n}\right)$. Then $\int_{0}^{t} f(\tau, \omega) d W_{\tau}$ is a martingale and

$$
\mathbb{E}\left[\sup _{0 \leq t \leq T}\left|\int_{0}^{T} f(\tau, \omega) d W_{\tau}\right|^{p}\right] \leq \frac{p}{p-1} \mathbb{E}\left[\left|\int_{0}^{T} f(\tau, \omega) d W_{\tau}\right|^{p}\right] .
$$

2.4. Short Time Existence. We now set up a contraction to show existence of a solution to (2.19) for a short time. Let $F: \mathbf{C}_{k, T}^{N} \times \mathbb{R}^{n} \times \mathbb{H}_{k}^{N} \rightarrow \mathbf{C}_{k, T}^{N}$ be defined by

$$
F(U, X, u)(t)=K_{t}(u)+\int_{0}^{t} K_{t-\tau}\left(f(U)+g \circ \mathcal{T}_{X_{\tau}}\right) d \tau
$$

and $G: \mathbf{C}_{k, t} \times \mathbb{R}^{n} \rightarrow \mathbb{R}^{n}$ be defined by

$$
G(U, X, \xi)(t)=\xi+\int_{0}^{t} a\left(U\left(X_{\tau}, \tau\right), X_{\tau}\right) d \tau+\int_{0}^{t} b\left(X_{\tau}\right) d W_{\tau}
$$


Then define

$$
H(U, X, u, \xi)(t)=(F(U, X, u)(t), G(U, X, \xi)(t))
$$

for $X, \xi \in \mathbb{R}^{n}, U \in \mathbf{C}_{k, T}^{N}$ and $u \in \mathbb{H}_{k}^{N}$.

To show $H$ is a contraction, we first let $0 \leq m_{1} \leq M_{1}<\infty, 0 \leq m_{2} \leq M_{2}<\infty$, and $0 \leq m_{3} \leq M_{3}<\infty$. For an integer $k \geq 4$, assume that $u \in \mathbf{B}_{k}^{N}\left(m_{1}, m_{2}\right),|\xi| \leq m_{3}$ and $(\bar{U}, X),(V, Y) \in \mathcal{C}_{k, T}^{N}\left(M_{1}, M_{2}, M_{3}\right)$. Then by $(2.22)$,

$$
\begin{aligned}
\sup _{0 \leq t \leq T}|F(U, X, u)| & =\sup _{0 \leq t \leq T}\left|K_{t}(u)+\int_{0}^{t} K_{t-\tau}(f(U)) d \tau+\int_{0}^{t} K_{t-\tau}(g) d \tau\right| \\
& \leq\left|K_{t}(u)\right|+\sup _{0 \leq t \leq T}\left|\int_{0}^{t} K_{t-\tau}(f(U)) d \tau\right|+\sup _{0 \leq t \leq T}\left|\int_{0}^{t} K_{t-\tau}(g) d \tau\right| \\
& \leq m_{1}+\sup _{0 \leq t \leq T} \int_{0}^{t}\left|K_{t-\tau}(f(U))\right| d \tau+\sup _{0 \leq t \leq T} \int_{0}^{t}\left|K_{t-\tau}(g)\right| d \tau \\
& \leq m_{1}+\int_{0}^{T}|f(U)| d \tau+\int_{0}^{T}|g| d \tau \\
& \leq m_{1}+T\left[c_{f, 1}\left(M_{1}\right) M_{1}+C_{g}\right] .
\end{aligned}
$$

Also by (2.23) and (2.30),

$$
\begin{aligned}
\|F(U, X, u)\|_{k, T} & =\sup _{0 \leq t \leq T}\left\|K_{t}(u)+\int_{0}^{t} K_{t-\tau}(f(U)) d \tau+\int_{0}^{t} K_{t-\tau}(g) d \tau\right\|_{k} \\
& \leq \sup _{0 \leq t \leq T}\left[\left\|K_{t}(u)\right\|_{k}+\left\|\int_{0}^{t} K_{t-\tau}(f(U)) d \tau\right\|_{k}+\left\|\int_{0}^{t} K_{t-\tau}(g) d \tau\right\|_{k}\right] \\
& \leq m_{2}+\sup _{0 \leq t \leq T} \int_{0}^{t}\left\|K_{t-\tau}(f(U))\right\|_{k} d \tau+\sup _{0 \leq t \leq T} \int_{0}^{t}\left\|K_{t-\tau}(g)\right\|_{k} d \tau \\
& \leq m_{2}+\int_{0}^{T}\|f(U)\|_{k} d \tau+\int_{0}^{T}\|g\|_{k} d \tau \\
& \leq m_{2}+T\left[C_{f, k}\left(M_{1}\right) M_{2}+C_{g, k}\right] .
\end{aligned}
$$

Finally,

$$
\begin{aligned}
\|G(U, X, \xi)\|_{\mathcal{S}_{T}}^{2} & =\mathbb{E}\left[\sup _{0 \leq t \leq T}|G(U, X, \xi)(t)|^{2}\right] \\
& =\mathbb{E}\left[\sup _{0 \leq t \leq T}\left|\xi+\int_{0}^{t} a\left(U\left(X_{s}, s\right), X_{s}\right) d s+\int_{0}^{t} b\left(X_{s}\right) d W_{s}\right|^{2}\right] \\
& \leq 3|\xi|^{2}+3 \mathbb{E}\left[\sup _{0 \leq t \leq T}\left(\left|\int_{0}^{t} a\left(U\left(X_{s}, s\right), X_{s}\right) d s\right|^{2}+\left|\int_{0}^{t} b\left(X_{s}\right) d W_{s}\right|^{2}\right)\right] \\
& \leq 3 m_{3}^{2}+3 \mathbb{E}\left[\sup _{0 \leq t \leq T} t \int_{0}^{t}\left|a\left(U\left(X_{s}, s\right), X_{s}\right) d s\right|^{2}+4\left|\int_{0}^{T} b\left(X_{s}\right) d W_{s}\right|^{2}\right]
\end{aligned}
$$

by the Cauchy-Schwarz inequality and Doob's martingale inequality. Then by the Itô Isometry,

$$
\|G(U, X, \xi)(t)\|_{\mathcal{S}_{T}}^{2} \leq 3\left(m_{3}+T^{2} C_{a}\left(M_{3}\right)+4 C_{b} T\right)
$$


Therefore, we can also choose $T$ such that

$$
\sup _{0 \leq t \leq T}|F(U, X, u)| \leq M_{1}, \quad\|F(U, X, u)\|_{k, T} \leq M_{2}, \quad \text { and } \quad\|G(U, X, \xi)\|_{\mathcal{S}_{T}} \leq M_{3} .
$$

Thus, $H$ takes $\mathcal{C}_{k, T}^{N}\left(M_{1}, M_{2}, M_{3}\right) \times \mathbf{B}_{k}^{N}\left(m_{1}, m_{2}\right) \times B_{m_{3}}(0)$ into $\mathcal{C}_{k, T}^{N}\left(M_{1}, M_{2}, M_{3}\right)$.

Next we show that $H$ is Lipschitz with respect to the norm $\|\cdot \cdot\|_{k, T}$. By (2.24),

$$
\begin{aligned}
& \|F(U, X, u)(t)-F(V, Y, u)(t)\|_{k} \\
& \quad=\left\|\int_{0}^{t} K_{t-\tau}(f(U(\tau))-f(V(\tau))) d \tau+\int_{0}^{t}\left(g \circ \mathcal{T}_{X_{\tau}}-g \circ \mathcal{T}_{Y_{\tau}}\right) d \tau\right\|_{k} \\
& \quad \leq \int_{0}^{t}\|f(U(\tau))-f(V(\tau))\|_{k} d \tau+\int_{0}^{t}\left\|g \circ \mathcal{T}_{X_{\tau}}-g \circ \mathcal{T}_{Y_{\tau}}\right\|_{k} d \tau \\
& \quad \leq L_{f, k}\left(M_{1}, M_{2}\right) \int_{0}^{t}\|U(\tau)-V(\tau)\|_{k} d \tau+L_{g, k} \int_{0}^{t}\left|X_{\tau}-Y_{\tau}\right| d \tau .
\end{aligned}
$$

By the Cauchy-Schwarz inequality,

$$
\begin{aligned}
& \mathbb{E}\left[\|F(U, X, u)-F(V, Y, u)\|_{k, T}^{2}\right] \\
& =\mathbb{E}\left[\sup _{0 \leq t \leq T}\left(L_{f, k}\left(M_{1}, M_{2}\right) \int_{0}^{t}\|U(\tau)-V(\tau)\|_{k} d \tau+L_{g, k} \int_{0}^{t}\left|X_{\tau}-Y_{\tau}\right| d \tau\right)^{2}\right] \\
& \leq 2 \mathbb{E}\left[\sup _{0 \leq t \leq T} L_{f, k}\left(M_{1}, M_{2}\right)^{2}\left(\int_{0}^{t}\|U(\tau)-V(\tau)\|_{k} d \tau\right)^{2}\right. \\
& \left.\quad+L_{g, k}^{2}\left(\int_{0}^{T}\left|X_{\tau}-Y_{\tau}\right| d \tau\right)^{2}\right] \\
& \leq 2 T \mathbb{E}\left[L_{f, k}\left(M_{1}, M_{2}\right)^{2} \int_{0}^{T}\|U(\tau)-V(\tau)\|_{k}^{2} d \tau+L_{g, k}^{2} \int_{0}^{T}\left|X_{\tau}-Y_{\tau}\right|^{2} d \tau\right] \\
& \leq 2 T^{2} \mathbb{E}\left[L_{f, k}\left(M_{1}, M_{2}\right)^{2}\|U(\tau)-V(\tau)\|_{k}^{2}+L_{g, k}^{2} \sup _{0 \leq t \leq T}\left|X_{\tau}-Y_{\tau}\right|^{2}\right] \\
& \leq T^{2} L_{F, k}\left(M_{1}, M_{2}\right)^{2}\|(U, X)-(V, Y)\|_{k, T}^{2}
\end{aligned}
$$

where $L_{F}\left(M_{2}\right)^{2}=2 \max \left\{L_{f, k}^{2}\left(M_{2}\right), L_{g, k}\right\}$. Define

$$
\begin{aligned}
\tilde{a}\left(U(t), X_{t}, V(t), Y_{t}\right) & =a\left(U\left(X_{t}, t\right), X_{t}\right)-a\left(V\left(Y_{t}, t\right), Y_{t}\right), \\
\tilde{b}\left(X_{t}, Y_{t}\right) & =b\left(X_{t}\right)-b\left(Y_{t}\right) .
\end{aligned}
$$

Then by Lemma 2.16,

$$
\begin{aligned}
& \|G(U, X, \xi)(t)-G(V, Y, \xi)(t)\|_{\mathcal{S}_{T}}^{2}=\mathbb{E}\left[\sup _{0 \leq t \leq T}|G(U, X, \xi)(t)-G(V, Y, \xi)(t)|^{2}\right] \\
& \quad=\mathbb{E}\left[\sup _{0 \leq t \leq T}\left|\int_{0}^{t} \tilde{a}\left(U(\tau), X_{\tau}, V(\tau), Y_{\tau}\right) d \tau+\int_{0}^{t} \tilde{b}\left(X_{\tau}, Y_{\tau}\right) d W_{\tau}\right|^{2}\right] \\
& \quad \leq 2 \mathbb{E}\left[\sup _{0 \leq t \leq T}\left|\int_{0}^{t} \tilde{a}\left(U(\tau), X_{\tau}, V(\tau), Y_{\tau}\right) d \tau\right|^{2}+\sup _{0 \leq t \leq T}\left|\int_{0}^{t} \tilde{b}\left(X_{\tau}, Y_{\tau}\right) d W_{\tau}\right|^{2}\right]
\end{aligned}
$$




$$
\begin{aligned}
& \leq 2 \mathbb{E}\left[\sup _{0 \leq t \leq T} t \int_{0}^{T}\left|\tilde{a}\left(U(\tau), X_{\tau}, V(\tau), Y_{\tau}\right)\right|^{2} d \tau+4\left|\int_{0}^{T} \tilde{b}\left(X_{\tau}, Y_{\tau}\right) d W_{\tau}\right|^{2}\right] \\
& \leq 2 \mathbb{E}\left[T \int_{0}^{T}\left|\tilde{a}\left(U(\tau), X_{\tau}, V(\tau), Y_{\tau}\right)\right|^{2} d \tau+4 \int_{0}^{T}\left|\tilde{b}\left(X_{\tau}, Y_{\tau}\right)\right|^{2} d \tau\right] \\
& \leq 2 T \mathbb{E}\left[T L_{a, 1}\left(M_{2}\right)\|U(t)-V(t)\|_{k}+\left(T L_{a, 2}\left(M_{2}\right)^{2}+4 L_{b}^{2}\right) \sup _{0 \leq t \leq T}\left|X_{t}-Y_{t}\right|^{2}\right] \\
& \leq T L_{G, k}\left(M_{2}, M_{3}\right)^{2}\|(U, X)-(V, Y)\|_{k, T}^{2}
\end{aligned}
$$

where $\left.L_{G, k}\left(M_{2}, M_{3}\right)^{2}=2 \max \left\{T L_{a, 1}\left(M_{2}\right), T L_{a, 2}\left(M_{2}\right)^{2}+4 L_{b}^{2}\right)\right\}$. Thus,

$$
\|H(U, X, u, \xi)-H(V, Y, u, \xi)\|_{k, T}^{2} \leq L_{H, k}^{2}\|(U, X)-(V, Y)\|_{k, T}^{2}
$$

where $L_{H, k}^{2}=L_{H, k}\left(M_{1}, M_{2}, M_{3}\right)^{2}=T \max \left\{T L_{F, k}\left(M_{1}, M_{2}\right)^{2}, L_{G, k}\left(M_{2}, M_{3}\right)^{2}\right\}$. Thus, $H$ is a contraction for $T<L_{H, k}\left(M_{1}, M_{2}, M_{3}\right)^{-1}$. Given $(U, X)(0)=(u, \xi)$ it follows that there is a unique $(U, X) \in \mathcal{C}_{k, T}^{N}\left(M_{1}, M_{2}, M_{3}\right)$ such that $H(U, X, u, \xi)=(U, X)$.

REMARK 2.20. Let $k \geq 0,(U, X),(V, Y) \in \mathcal{C}_{4, T}^{N}\left(M_{1}, M_{2}, M_{3}\right)$ and $(u, \xi) \in$ $\mathcal{B}_{4}^{N}\left(M_{1}, M_{2}, M_{3}\right)$. Using the same argument as above, we also have the estimate

$$
\|H(U, X, u, \xi)-H(V, Y, u, \xi)\|_{k, T}^{2} \leq L_{H, k \vee 3}\|(U, X)-(V, Y) \mid\|_{k \vee 3, T} .
$$

The next lemma is the basis of the error estimates that follow.

LEMma 2.21. Let $k$ be a nonnegative integer, $(U, X),(V, Y) \in \mathcal{C}_{4, T}^{N}\left(M_{1}, M_{2}, M_{3}\right)$ and $H(U, X, u, \xi)=(U, X)$. Then

$$
\left\|(U, X)-(V, Y)\left|\left\|_{k, T} \leq \frac{1}{1-L_{H, k}} \mid\right\| H(V, Y, u, \xi)-(V, Y) \|_{k \vee 3, T}\right.\right.
$$

provided that $L_{H, k}=L_{H, k}\left(M_{1}, M_{2}, M_{3}\right)<1$.

Proof. Since $H(U, X, u, \xi)=(U, X)$,

$$
\begin{aligned}
& \|(U, X)-(V, Y)\|\left\|_{k, T}=\right\| H(U, X, u, \xi)-H(V, Y, u, \xi)+H(V, Y, u, \xi)-(V, Y)\|\|_{k, T} \\
& \quad \leq\|H(U, X, u, \xi)-H(V, Y, u, \xi)\|\left\|_{k, T}+\right\| H(V, Y, u, \xi)-(V, Y)\|\|_{k, T} \\
& \quad \leq L_{H, k}\left(M_{1}, M_{2}, M_{3}\right)\|(U, X)-(V, Y)\|_{k \vee 3, T}+\|H(V, Y, u, \xi)-(V, Y)\| \|_{k \vee 3, T}
\end{aligned}
$$

from which (2.42) immediately follows.

3. Operator Splitting. In this section we will apply operator splitting to the reaction-diffusion stochastic system (2.19). This will aid in both proving the existence of long time solutions and solving the system numerically.

3.1. Taylor Expansions. In order to analyze the error due to operator splitting, we introduce some notation. Suppose $X$ and $Y$ are normed vector spaces. Let $f$ be a $J$ times continuously differentiable function mapping an open convex subset $A$ of $X$ into $Y$. For each $j=0,1, \ldots, J$ we let $f^{[j]}$ be the $j$-th differential of $f$. Thus, for each $a \in A, f^{(j)}(a)$ is a $j$ th degree polynomial function on $X$ whose value at $v \in X$ is

$$
\left.\left(\frac{d}{d t}\right)^{j} f(a+t v)\right|_{t=0}
$$


Let $f^{\{J\}}$ be the function on $A \times A$ whose value at $(a, b) \in A \times A$ is the $J$-th degree polynomial function on $X$ whose value at $v \in X$ is

$$
\int_{0}^{1} f^{[j]}\left(a+\lambda(b-a)(v) d(1-\lambda)^{N} .\right.
$$

Taylor's Theorem implies that

$$
f(b)=\sum_{j=0}^{J-1} f^{[j]}(a)(b-a)+\frac{1}{J !} f^{\{J\}}(a, b)(b-a), \quad(a, b) \in A \times A .
$$

Suppose $B$ is an open subset of $X \times[0, \infty)$ with the property that if $b \in B$ then $\{t:(b, t) \in B\}$ is an interval containing 0 . Let $f$ be a $J$ times continuously differentiable function mapping $B$ into $Y$. For each $j=0,1, \ldots, J$ let

$$
f^{(j)}(b)=\left.\left(\frac{d}{d t}\right)^{j} f(b, t)\right|_{t=0}, \quad b \in B
$$

and

$$
f^{<J>}(b, t)=\int_{0}^{1}\left(\frac{d}{d \lambda}\right)^{J} f(\lambda b) d(1-\lambda)^{N}
$$

Then Taylor's Theorem implies that

$$
f(b, t)=\sum_{j=0}^{J-1} \frac{t^{j}}{j !} f^{(j)}(b)+\frac{t^{J}}{J !} f^{<J>}(b, t)
$$

3.2. Ordinary Differential Equations. Let $f: \mathbb{R}^{N} \rightarrow \mathbb{R}^{N}$ be smooth. Suppose $R$ is the flow of $f$, i.e. the set of $\left(\left(t, u_{0}\right), u_{1}\right) \in \mathbb{R} \times \mathbb{R}^{N}$ such that either $t=0$ and $u_{0}=u_{1}$ or $t \neq 0$ and there is a continuously differentiable function $R$ carrying an open interval $I$ with $\{0, t\} \subset I$ into $\mathcal{G}$ such that

$$
\frac{d}{d \tau} R(\tau)=f(R(\tau)) \quad \text { for } \tau \in I
$$

It is well known that $R$ is a function; the domain of $R$ is an open subset of $\mathbb{R} \times \mathbb{R}^{N}$ which contains $\{0\} \times \mathbb{R}^{N}$; and that $R$ is smooth. We shall assume for convenience that the domain of $R$ is all of $\mathbb{R} \times \mathbb{R}^{N}$; it is a straightforward matter to modify what follows to obtain essentially the same results if this is not the case. It follows that

$$
R(0, u)=u, \quad \frac{\partial}{\partial t} R(t, u)=f(R(t, u)) \quad \text { whenever }(t, u) \in \mathcal{G} .
$$

It is easy to show that

$$
R^{(0)}(u)=u, \quad R^{(1)}(u)=f(u), \quad R^{(2)}(u)=f^{[1]}(u)(f(u)), \quad u \in \mathbb{R}^{N} .
$$

We shall abbreviate $R(t, u)$ by $R_{t}(u)$. 
3.3. A First Order Split Scheme. We can decouple the system (2.19) into three separate systems for the diffusion, reaction and motion of the cells, i.e.

$$
\begin{aligned}
\frac{d U}{d t} & =\mathcal{D}(U)+g \circ \mathcal{T}_{\xi}, & & U(0)=u, \\
\frac{d U}{d t} & =f(U), & & U(0)=u, \\
d X_{t} & =a\left(u, X_{t}\right) d t+b\left(X_{t}\right) d W_{t}, & X(0) & =\xi .
\end{aligned}
$$

Let $F, G$ and $H$ be defined by (2.37), (2.38) and (2.39), respectively. Let $K_{t}$ be defined by (2.10) and define

$$
L_{t}(u, \xi)=K_{t}(u)+\int_{0}^{t} K_{t-\tau}\left(g \circ \mathcal{T}_{\xi}\right) d \tau
$$

It follows from (2.11) that $L_{t}(u, \xi)$ solves the inhomogeneous diffusion equation (3.3). Then let

$$
M_{t}\left(\begin{array}{l}
u \\
\xi
\end{array}\right)=\left(\begin{array}{c}
L_{t}(u, \xi) \\
\xi
\end{array}\right)
$$

Let $R_{t}$ be the flow of (3.4) as defined by (3.1) and

$$
S_{t}\left(\begin{array}{l}
u \\
\xi
\end{array}\right)=\left(\begin{array}{c}
R_{t}(u, \xi) \\
\xi
\end{array}\right) .
$$

Finally, let $Y_{t}(u, \xi)$ be the solution of (3.5) given by

$$
Y_{t}(\xi, u)=\xi+\int_{0}^{t} a\left(u, Y_{\tau}\right) d \tau+\int_{0}^{t} b\left(Y_{\tau}\right) d W_{\tau}
$$

and define

$$
Z_{t}\left(\begin{array}{l}
u \\
\xi
\end{array}\right)=\left(\begin{array}{c}
u \\
Y_{t}(u, \xi)
\end{array}\right)
$$

Then a split-step method to approximate $(2.19)$ is

$$
\Psi_{t}(u, \xi)=Z_{t}\left(S_{t}\left(M_{t}(u, \xi)\right) .\right.
$$

The next theorem shows that the local truncation error for the split scheme $\Psi_{t}$ is $\mathcal{O}\left(t^{\frac{3}{2}}\right)$. In $\S 4$ we will show that the global error is $\mathcal{O}(t)$.

Definition 3.1. For $\phi=(u, \xi) \in \mathcal{B}_{k}\left(m_{1}, m_{2}, m_{3}\right)$ define

$$
|\phi|_{k}=\left|\mathbb{E}\left[\|u\|_{k}+\xi\right]\right| \text {. }
$$

TheOREM 3.2. Let $(U, X)$ be the solution of $(2.19)$ on $[0, T)$ where $(U, X)(0)=$ $(u, \xi) \in \mathbf{C}_{k, T}^{N}$. Let the error due to the split scheme (3.8) be given by

$$
\mathbf{e}(t)=(U, X)(t)-\Psi_{t}(u, \xi) .
$$

Then for a nonnegative integer $k$,

$$
\sup _{0 \leq t \leq T}|\mathbf{e}(\mathbf{t})|_{k} \leq C\left(\sup |u|,\|u\|_{(k+2) \vee 3}\right) T^{2} .
$$


and

$$
\|\mathbf{e} \mid\|_{k, T} \leq C\left(\sup |u|,\|u\|_{(k+2) \vee 3}\right) T^{\frac{3}{2}}
$$

Proof. Using Lemma 2.21, we estimate the error for the splitting $\Psi$ by estimating the difference between $H\left(\Psi_{t}(u, \xi), u, \xi\right)$ and $\Psi_{t}(u, \xi)$, i.e

$$
\begin{aligned}
\|\mathbf{e}(t)\| \|_{k, T} & \leq\left\|H\left(\Psi_{t}(u, \xi), u, \xi\right)-\Psi_{t}(u, \xi)\right\|_{k \vee 3, T} \\
& =\mathbb{E}\left[\sup _{0 \leq t \leq T}\left\{\left\|\mathbf{e}_{1}(t)\right\|_{k \vee 3}^{2}+\left|\mathbf{e}_{2}(t)\right|^{2}\right\}\right]^{\frac{1}{2}}
\end{aligned}
$$

where

$$
\begin{aligned}
& \mathbf{e}_{1}(t)=F\left(\Psi_{t}\left(\begin{array}{l}
u \\
\xi
\end{array}\right), u\right)-R_{t}\left(L_{t}(u, \xi)\right) \\
& \mathbf{e}_{2}(t)=G\left(\Psi_{t}\left(\begin{array}{l}
u \\
\xi
\end{array}\right), \xi\right)-Y_{t}\left(R_{t}\left(L_{t}(u, \xi)\right), \xi\right) .
\end{aligned}
$$

We immediately see from the definitions of $G$ and $Y_{t}$ that $\mathbf{e}_{2}(t)=0$.

We shall now examine $\mathbf{e}_{1}(t)$. By the definition of $F$,

$$
\begin{aligned}
\mathbf{e}_{1}(t)= & K_{t}(u)+\int_{0}^{t} K_{t-\tau} f\left(R_{\tau}\left(L_{\tau}(u, \xi)\right)\right) d \tau \\
& +\int_{0}^{t} K_{t-\tau}\left(g \circ \mathcal{T}_{Y_{\tau}\left(R_{\tau}\left(L_{\tau}(u, \xi)\right), \xi\right)}\right) d \tau-R_{t}\left(L_{t}(u, \xi)\right) .
\end{aligned}
$$

We proceed using Taylor expansions. A second order expansion of $R_{t}$ is

$$
\begin{aligned}
R_{t}\left(L_{t}(u, \xi)\right)= & L_{t}(u, \xi)+t R_{t}^{(1)}\left(L_{t}(u, \xi)\right)+\frac{t^{2}}{2} R^{<2>}\left(t, L_{t}(u, \xi)\right) \\
= & K_{t}(u)+\int_{0}^{t} K_{t-\tau}\left(g \circ \mathcal{T}_{Y_{\tau}(u, \xi)}\right) d \tau \\
& +t f\left(L_{t}(u, \xi)\right)+\frac{t^{2}}{2} R^{<2>}\left(t, L_{t}(u, \xi)\right)
\end{aligned}
$$

Substituting the above expansion,

$$
\mathbf{e}_{1}(t)=\mathbf{e}_{11}(t)+\mathbf{e}_{12}(t)-\frac{t^{2}}{2} R^{<2>}\left(t, L_{t}(u, \xi)\right) .
$$

where

$$
\begin{aligned}
& \mathbf{e}_{11}(t)=\int_{0}^{t} K_{t-\tau} f\left(R_{\tau}\left(L_{\tau}(u, \xi)\right)\right) d \tau-t f\left(L_{t}(u, \xi)\right), \\
& \mathbf{e}_{12}(t)=\int_{0}^{t} K_{t-\tau}\left(g \circ \mathcal{T}_{Y_{\tau}\left(R_{\tau}\left(L_{\tau}(u, \xi)\right), \xi\right)}\right) d \tau-\int_{0}^{t} K_{t-\tau}\left(g \circ \mathcal{T}_{\xi}\right) d \tau .
\end{aligned}
$$

A first order Taylor expansion of $f$ is given by

$$
f(x+h)=f(x)+f^{\{1\}}(x, x+h)(h) .
$$


If we substitute a first order Taylor expansion of $R_{\tau}$ in $f\left(R_{\tau}\left(L_{\tau}(u, \xi)\right)\right)$, then

$$
\begin{aligned}
& f\left(R_{\tau}\left(L_{\tau}(u, \xi)\right)\right)=f\left(L_{\tau}(u, \xi)\right) \\
& \quad+f^{\{1\}}\left(L_{\tau}(u, \xi), R_{\tau}\left(L_{\tau}(u, \xi)\right)\left(\tau R^{<1>}\left(\tau, L_{\tau}(u, \xi)\right)\right)\right.
\end{aligned}
$$

Thus,

$$
\mathbf{e}_{11}(t)=\int_{0}^{t} K_{t-\tau} f\left(L_{\tau}(u, \xi)\right) d \tau-t f\left(L_{t}(u, \xi)\right)+\mathcal{R}_{t}(u, \xi),
$$

where the remainder term

$$
\mathcal{R}_{t}(u, \xi)=\int_{0}^{t} K_{t-\tau} f^{\{1\}}\left(L_{\tau}(u, \xi), R_{\tau}\left(L_{\tau}(u, \xi)\right)\left(\tau R^{<1>}\left(\tau, L_{\tau}(u, \xi)\right)\right) d \tau\right.
$$

is $\mathcal{O}\left(t^{2}\right)$. Let $\eta(\tau)=K_{t-\tau} f\left(L_{\tau}(u, \xi)\right)$. Then

$$
\begin{aligned}
\left\|\mathbf{e}_{11}(t)\right\|_{k} & =\left\|\int_{0}^{t} \eta(\tau) d \tau-t \eta(t)\right\|_{k} \leq \int_{0}^{t}\|\eta(\tau)-\eta(t)\|_{k} d \tau \\
& \leq \int_{0}^{t}\left\|\eta^{\prime}(\tau)\right\|_{k}|t-\tau| d \tau=\sup _{0 \leq \tau \leq t}\left\|\eta^{\prime}(\tau)\right\|_{k} \frac{t^{2}}{2} .
\end{aligned}
$$

Then by Theorem 2.13 and Lemma 2.15,

$$
\begin{aligned}
\left\|\eta^{\prime}(\tau)\right\|_{k} & =\left\|\frac{\partial}{\partial \tau} K_{t-\tau} f\left(L_{\tau}(u, \xi)\right)\right\|_{k}=\left\|\mathcal{D} K_{t-\tau} f\left(L_{\tau}(u, \xi)\right)\right\|_{k} \\
& \leq\left\|f\left(L_{\tau}(u, \xi)\right)\right\|_{k+2} \leq C_{f, k}(\sup |u|)\left\|L_{\tau}(u, \xi)\right\|_{k+2} \\
& \leq C_{f, k}(\sup |u|)\left\|K_{t}(u)+\int_{0}^{t} K_{t-\tau}\left(g \circ \mathcal{T}_{\xi}\right) d \tau\right\|_{k+2} \\
& \leq C_{f, k}(\sup |u|)\left(\|u\|_{k+2}+C_{g, k+2}\right) .
\end{aligned}
$$

Therefore,

$$
\left\|\mathbf{e}_{11}\right\|_{k} \leq C_{f, k}\left(\sup |u|,\|u\|_{k+2}\right) t^{2}
$$

Using (2.12) and Corollary 2.15,

$$
\begin{aligned}
\left\|\mathbf{e}_{12}(t)\right\|_{k} & \leq \int_{0}^{t}\left\|K_{t-\tau}\left(g \circ \mathcal{T}_{Y_{\tau}\left(R_{\tau}\left(L_{\tau}(u, \xi)\right), \xi\right)}-g \circ \mathcal{T}_{Y_{\tau}(u, \xi)}\right)\right\|_{k} d \tau \\
& \leq \int_{0}^{t}\left\|g \circ \mathcal{T}_{Y_{\tau}\left(R_{\tau}\left(L_{\tau}(u, \xi)\right), \xi\right)}-g \circ \mathcal{T}_{\xi}\right\|_{k} d \tau \\
& \leq L_{g, k} \int_{0}^{t}\left|Y_{\tau}\left(R_{\tau}\left(L_{\tau}(u, \xi)\right), \xi\right)-\xi\right| d \tau . \\
& \leq L_{g, k} \int_{0}^{t} \int_{0}^{\tau}\left|a\left(R_{s}\left(L_{s}(u, \xi)\right), \xi\right)\right| d s d \tau+\int_{0}^{t}\left|\int_{0}^{\tau} b(\xi) d W_{s}\right| d \tau
\end{aligned}
$$

Since the expectation of the Itô integral is always zero,

$$
\begin{aligned}
\mathbb{E}\left[\sup _{0 \leq t \leq T}\left\|\mathbf{e}_{12}(t)\right\|_{k}\right] \leq & L_{g, k} \mathbb{E}\left[\sup _{0 \leq t \leq T} \int_{0}^{t} \int_{0}^{\tau}\left|a\left(R_{s}\left(L_{s}(u, \xi)\right), \xi\right) d s d \tau\right|\right] \\
& +L_{g, k} \mathbb{E}\left[\sup _{0 \leq t \leq T} \int_{0}^{t}\left|\int_{0}^{\tau} b(\xi) d W_{s}\right| d \tau\right] \\
\leq & \frac{1}{4} L_{g, k} C_{a}(\xi) T^{2}
\end{aligned}
$$


By the Cauchy-Schwarz Inequality, Doob's martingale inequality and the Itô Isometry,

$$
\begin{aligned}
\mathbb{E}\left[\sup _{0 \leq t \leq T}\left\|\mathbf{e}_{12}(t)\right\|_{k}^{2}\right] \leq & 2 L_{g, k}\left(\mathbb{E}\left[\sup _{0 \leq t \leq T}\left(\int_{0}^{t} \int_{0}^{\tau}\left|a\left(R_{s}\left(L_{s}(u, \xi)\right), \xi\right) d s d \tau\right|\right)^{2}\right]\right) \\
& +\mathbb{E}\left[\sup _{0 \leq t \leq T}\left(\int_{0}^{t}\left|\int_{0}^{\tau} b(\xi) d W_{s}\right| d \tau\right)^{2}\right] \\
\leq & 2 L_{g, k}\left(\frac{1}{4} C_{a}^{2}(\xi) T^{4}+4 T \mathbb{E}\left[\sup _{0 \leq t \leq T} \int_{0}^{t} \int_{0}^{\tau}|b(\xi)|^{2} d s d \tau\right]\right) \\
\leq & 2 L_{g, k}\left(\frac{1}{4} C_{a}^{2}(\xi) T^{4}+2 C_{b}^{2} T^{3}\right)
\end{aligned}
$$

Combining (3.12) and (3.13),

$$
\mathbb{E}\left[\sup _{0 \leq t \leq T}\left\|\mathbf{e}_{1}(t)\right\|_{k}\right] \leq C\left(\sup |u|,\|u\|_{(k+2)}\right) T^{2} .
$$

From (3.12) and (3.14) we have

$$
\mathbb{E}\left[\sup _{0 \leq t \leq T}\left\|\mathbf{e}_{1}(t)\right\|_{k}^{2}\right]^{\frac{1}{2}} \leq C\left(\sup |u|,\|u\|_{(k+2)}\right) T^{\frac{3}{2}} .
$$

Equations (3.10) and (3.11) follow from (3.15), (3.16) and the fact that $\mathbf{e}_{2}(t)=0$.

4. Convergence and Long Time Existence. In this section we will prove long time existence by first showing that the splitting outlined in Section 4 converges to the true solution. As a consequence, the reaction-diffusion equations preserve positivity. It follows that the supremum of the solution is a priori bounded and its derivatives are bounded for finite time. Assuming the solutions to (2.19) exists for $t \in[0, T)$, the limit as $t$ approaches $T$ also exists, allowing us to solve (2.19) for further time.

4.1. Preliminaries. Suppose $k \geq 4, t \in[0, \infty)$ and $\phi=(u, \xi) \in \mathcal{B}_{k}^{N}\left(m_{1}, m_{2}, m_{3}\right)$ for $0 \leq m_{1}, m_{2}, m_{3}<\infty$. Let

$$
\Phi(t, \phi, \delta)=(U(t, \phi, \delta), X(t, \phi, \delta))
$$

be the flow of the reaction-diffusion-stochastic system (2.19), i.e. the set of $((t, \phi, \tau), \eta)$ such that there exists a solution $\mathcal{U}$ of (2.19) whose domain is an interval containing $t$ and $t+\tau, \mathcal{U}(t)=\phi$ and $\mathcal{U}(t+\tau)=\eta$. It is a consequence of uniqueness that $\Phi$ is a function. By local existence, for any $(t, \phi) \in[0, \infty) \times \mathcal{B}_{k}^{N}\left(m_{1}, m_{2}, m_{3}\right)$, there exists $\tau>0$ such that $(t, \phi, \tau) \in$ domain $\Phi$. It also follows from uniqueness that if $\delta_{1}$ and $\delta_{2}$ are positive, then

$$
\Phi\left(t, \phi, \delta_{1}+\delta_{2}\right)=\Phi\left(t, \Phi\left(t, \phi, \delta_{1}\right), \delta_{2}\right) .
$$

Suppose $\Psi(t, \phi, \delta)=(V(t, \phi, \delta), Y(t, \phi, \delta))$ is a one step method for solving (2.19) such that $\Psi(t, \phi, 0)=\phi$. Then the local truncation error for the method $\Psi$ is given by

$$
T(t, \phi, \delta)=\Phi(t, \phi, \delta)-\Psi(t, \phi, \delta)=\left(T_{U}(t, \phi, \delta), T_{X}(t, \phi, \delta)\right)
$$

where

$$
\begin{aligned}
& T_{U}(t, \phi, \delta)=U(t, \phi, \delta)-V(t, \phi, \delta), \\
& T_{X}(t, \phi, \delta)=X(t, \phi, \delta)-Y(t, \phi, \delta) .
\end{aligned}
$$


Suppose $t_{0}, T \in[0, \infty)$ such that $t_{0}<T$. Let $J$ be a positive integer such that $\delta=\frac{T}{J}<1$. Then for each $j=1, \ldots, J$ let

$$
\begin{array}{lll}
t_{j}=t_{j-1}+\delta ; & & \\
\Phi_{j}=\Phi\left(t_{0}, \Phi_{0}, t_{j}-t_{0}\right) ; & U_{j}=U\left(t_{0}, U_{0}, t_{j}-t_{0}\right) ; & X_{j}=X\left(t_{0}, X_{0}, t_{j}-t_{0}\right) \\
\Psi_{j}=\Psi\left(t_{j-1}, \Psi_{j-1}, \delta\right) ; & V_{j}=V\left(t_{j-1}, V_{j-1}, \delta\right) ; & Y_{j}=Y\left(t_{j-1}, Y_{j-1}, \delta\right) \\
e_{j}=\Phi_{j}-\Psi_{j} ; & & \\
T_{j}=T\left(t_{j-1}, \Psi_{j-1}, \delta\right) & &
\end{array}
$$

Finally, define

$$
\Phi_{t, \phi}(x, \delta)=\Phi(t, \phi, \delta)(x) \quad \text { and } \quad \Psi_{t, \psi}(x, \delta)=\Psi(t, \psi, \delta)(x) .
$$

The next theorem which gives the error due to the difference in initial conditions is based on a lemma from [9].

Theorem 4.1. Let $Z=\left(Z_{U}, Z_{X}\right)$ be defined by

$$
\Phi(t, \phi, \delta)-\Phi(t, \psi, \delta)=\left(\begin{array}{c}
K_{\delta}(u-v) \\
\xi-\zeta
\end{array}\right)+\left(\begin{array}{c}
Z_{U} \\
Z_{X}
\end{array}\right)
$$

where $\phi=(u, \xi), \psi=(v, \zeta)$. Then for any integer $k \geq 4$ there exists a constant $C_{\Phi, k}=C\left(\sup |U|,\|U\|_{k},\|X\|_{\mathcal{S}_{T}}\right)$ which is independent of $\delta$ for $\delta<1$ such that

$$
\begin{gathered}
\left\|\Phi_{t, \phi}-\Phi_{t, \psi}\right\|_{k, \delta}^{2} \leq\|\phi-\psi \mid\|_{k}^{2}\left(1+C_{\Phi, k} \delta\right), \\
\|Z\|_{k, \delta}^{2} \leq C_{\Phi, k}\|\phi-\psi\|_{k}^{2} \delta .
\end{gathered}
$$

Before we prove this theorem, we must first state several lemmas.

Lemma 4.2. Let $\mathbb{B}$ be a Banach space with norm $\|\cdot\|$ and $\mathbb{C}$ be the space of functions $\beta:[0, T) \rightarrow \mathbb{B}$ which are continuous with respect to the norm $\|\cdot\|$. Suppose $\alpha \in \mathbb{B}$ and $\beta \in \mathbb{C}$. Then

$$
\left\|\alpha+\int_{t}^{t+\delta} \beta(\tau) d \tau\right\|^{2} \leq(1+\delta)\|\alpha\|^{2}+(1+\delta) \int_{t}^{t+\delta}\|\beta(\tau)\|^{2} d \tau .
$$

Proof. By the triangle inequality and Cauchy-Schwarz inequality,

$$
\begin{aligned}
& \left\|\alpha+\int_{t}^{t+\delta} \beta(\tau) d \tau\right\|^{2} \leq\left(\|\alpha\|+\left\|\int_{t}^{t+\delta} \beta(\tau) d \tau\right\|\right)^{2} \\
& \quad \leq\|\alpha\|^{2}+2\|\alpha\|\left\|\int_{t}^{t+\delta} \beta(\tau) d \tau\right\|+\left\|\int_{t}^{t+\delta} \beta(\tau) d \tau\right\|^{2} \\
& \quad \leq\|\alpha\|^{2}+2 \delta^{\frac{1}{2}}\|\alpha\|\left(\int_{t}^{t+\delta}\|\beta(\tau)\|^{2} d \tau\right)^{\frac{1}{2}}+\delta \int_{t}^{t+\delta}\|\beta(\tau)\|^{2} d \tau \\
& \quad \leq(1+\delta)\|\alpha\|^{2}+(1+\delta) \int_{t}^{t+\delta}\|\beta(\tau)\|^{2} d \tau .
\end{aligned}
$$


Corollary 4.3. For $\alpha \in \mathbb{B}$ and $\beta, \gamma \in \mathbb{C}$,

$$
\begin{aligned}
\left\|\alpha+\int_{t}^{t+\delta}(\beta(\tau)+\gamma(\tau)) d \tau\right\|^{2} \leq(1 & +\delta)\|\alpha\|^{2} \\
& +2(1+\delta) \int_{t}^{t+\delta}\left(\|\beta(\tau)\|^{2}+\|\gamma(\tau)\|^{2}\right) d \tau .
\end{aligned}
$$

The next lemma from [9] is easily proved using induction.

Lemma 4.4. Let $A, B, \delta>0$ and $p \geq 1$. If

$$
\mu_{j+1} \leq(1+A \delta) \mu_{j}+B \delta^{p},
$$

then

$$
\mu_{j} \leq e^{A T} \mu_{0}+\frac{B}{A}\left(e^{A T}-1\right) \delta^{p-1}
$$

Proof. [Theorem 4.1] We will consider the two components of $\Phi=(U, X)$ separately. In the context of Corollary 4.3 , let $\alpha=K_{\delta}(u-v)$ and

$$
\int_{t}^{t+\delta}(\beta(\tau)+\gamma(\tau)) d \tau=Z_{U} .
$$

Then

$$
\begin{aligned}
& \|U(t, \phi, \delta)-U(t, \psi, \delta)\|_{k}^{2} \\
& \leq(1+\delta)\left\|K_{\delta}(u-v)\right\|_{k}^{2}+2(1+\delta) \int_{t}^{t+\delta}\left\|K_{t-\tau}[f(U(t, \phi, \tau))-f(U(t, \psi, \tau))]\right\|_{k}^{2} d \tau \\
& \quad+2(1+\delta) \int_{t}^{t+\delta}\left\|K_{t-\tau}[g(X(t, \phi, \tau))-g(X(t, \psi, \tau))]\right\|_{k}^{2} d \tau \\
& \leq(1+\delta)\|u-v\|_{k}^{2}+2(1+\delta) L_{f, k}\left(\sup |U|,\|U\|_{k}\right)^{2} \int_{t}^{t+\delta}\|U(t, \phi, \tau)-U(t, \psi, \tau)\|_{k}^{2} d \tau \\
& \quad+2(1+\delta) L_{g, k}^{2} \int_{t}^{t+\delta}|X(t, \phi, \tau)-X(t, \psi, \tau)|^{2} d \tau
\end{aligned}
$$

Define

$$
\begin{aligned}
& \tilde{a}(\tau)=a\left(U(t, \phi, \tau), X_{\tau}(t, \phi, \tau)\right)-a(U(t, \psi, \tau), X(t, \psi, \tau)), \\
& \tilde{b}(\tau)=b\left(X_{\tau}(t, \phi, \tau)\right)-b(X(t, \psi, \tau)) .
\end{aligned}
$$

Since the expected value of the Itô integral is zero,

$$
\begin{aligned}
& \mathbb{E}\left[\sup _{0 \leq s \leq \delta}|X(t, \phi, s)-X(t, \psi, s)|^{2}\right] \\
& \quad=\mathbb{E}\left[\sup _{0 \leq s \leq \delta}\left|\xi-\zeta+\int_{t}^{t+s} \tilde{a}(\tau) d \tau\right|^{2}\right]+\mathbb{E}\left[\sup _{0 \leq s \leq \delta}\left|\int_{t}^{t+s} \tilde{b}(\tau) d W_{\tau}\right|^{2}\right] .
\end{aligned}
$$


In the context of Lemma 4.2, let $\alpha=\xi-\zeta$ and $\beta=\tilde{a}$. Then

$$
\begin{aligned}
& \mathbb{E}\left[\sup _{0 \leq s \leq \delta}|X(t, \phi, s)-X(t, \psi, s)|^{2}\right] \\
& \leq(1+\delta) \mathbb{E}\left[|\xi-\zeta|^{2}\right]+(1+\delta) \mathbb{E}\left[\int_{t}^{t+\delta}|\tilde{a}(\tau)|^{2} d \tau\right]+4 \mathbb{E}\left[\int_{t}^{t+\delta}|\tilde{b}(\tau)|^{2} d \tau\right] \\
& \leq(1+\delta) \mathbb{E}\left[|\xi-\zeta|^{2}\right] \\
&+(1+\delta) L_{a, 1}\left(\|U\|_{k}\right)^{2} \int_{t}^{t+\delta} \mathbb{E}\left[\sup _{0 \leq \sigma \leq \tau}\|U(t, \phi, \sigma)-U(t, \psi, \sigma)\|_{k}^{2}\right] d \tau \\
&+(1+\delta) L_{a, 2}\left(\|U\|_{k},\|X\|_{\mathcal{S}_{T}}\right)^{2} \int_{t}^{t+\delta} \mathbb{E}\left[\sup _{0 \leq \sigma \leq \tau}|X(t, \phi, \sigma)-X(t, \psi, \sigma)|^{2}\right] d \tau \\
&+L_{b}^{2} \int_{t}^{t+\delta} \mathbb{E}\left[\sup _{0 \leq \sigma \leq \tau}|X(t, \phi, \sigma)-X(t, \psi, \sigma)|^{2}\right] d \tau
\end{aligned}
$$

by the Doob martingale inequality and the Itô Isometry. Therefore,

$$
\begin{aligned}
& \|\| \Phi(t, \phi, s)-\Phi(t, \psi, s)\left\|_{k, \delta}^{2} \leq(1+\delta)\right\| \phi-\psi \mid \|_{k}^{2} \\
& \quad+C_{\Phi, k}\left(\sup |U|,\|U\|_{k},\|X\|_{\mathcal{S}_{T}}\right) \int_{t}^{t+\delta}\|\Phi(t, \phi, \tau)-\Phi(t, \psi, \tau)\|_{k, \delta}^{2} d \tau
\end{aligned}
$$

where

$$
\begin{gathered}
C_{\Phi, k}\left(\sup |U|,\|U\|_{k},\|X\|_{\mathcal{S}_{T}}\right)=\max \left\{(1+\delta) \delta\left(2 L_{f, k}\left(\sup |U|,\|U\|_{k}\right)^{2}+L_{a, 1}\left(\|U\|_{k}\right)^{2}\right),\right. \\
(1+\delta)\left(2 L_{g, k}^{2}+L_{a, 2}\left(\|U\|_{k},\|X\|_{\mathcal{S}_{T}}\right)^{2}\right)+L_{b}^{2}
\end{gathered}
$$

Then by the Gronwall inequality,

$$
\left\|\Phi_{t, \phi}-\Phi_{t, \psi}\right\|_{k, \delta}^{2} \leq\|\phi-\psi\|_{k}^{2}(1+\delta) e^{C_{\Phi, k} \delta},
$$

Equation (4.7) immediately follows and (4.8) is the result of subtracting $\mathbb{E}\left[|\xi-\zeta|^{2}\right]$ from (4.15) and (4.17). $\square$

REMARK 4.5. The proof of this lemma can be modified to yield the same estimate with the $\|\cdot \mid\|_{k}$ norm instead of the $\|\cdot\| \cdot \|_{k, \delta}$ norm.

4.2. Positivity. It is well known that if the initial condition is nonnegative, i.e. $u_{i} \geq 0$ for $i=1,2, \ldots, N$, the solution to the diffusion (3.3) is positive, i.e for $t>0$, $U_{i}(t)>0$ for all $i$. The following lemma shows that if the initial condition is positive, the solution to the reaction (3.4) is also positive.

Lemma 4.6. Let $I$ be an open interval. Suppose $U: I \rightarrow \mathbb{R}^{n}$ satisfies (3.4) and for some $t_{0} \in I, U_{i}\left(t_{0}\right)>0$ for $i=1,2, \ldots, N$. Then for all $t \in I, U_{i}(t)>0$ for all $i$.

Proof. We first note that if $u_{i}=0$ for some $i$, then there exists a solution of (3.4) such that $U_{i}(t)=0$ for all $t>0$. Let $t_{0} \in I$ and $U: I \rightarrow \mathbb{R}^{n}$ be a trajectory for (3.4) such that $U_{i}\left(t_{0}\right)=0$ for some $i$. Then $f\left(U\left(t_{0}\right)\right)=0$ and it follows by uniqueness that $U_{i}(t)=0$ for all $t \in I$. Therefore if $U_{i}\left(t_{0}\right)>0$ for all $i$, for all $t \in I, U_{i}(t)>0$ for all $i$. $\mathrm{U}$

Since the split-step method for the reaction-diffusion equation converges to the true solution, the solution to the reaction diffusion equation is positive if the initial condition is nonnegative. 
4.3. Error Estimates for Numerical Methods. In this section we will show that the splitting outlined in chapter 3 is a first order method. These ideas can be extended to show the convergence rates of other numerical methods as well.

Throughout this section we assume that the solution of (2.19) exists on the interval $[0, T)$. We begin by showing that the splitting

$$
\left.\Psi_{t}(u, \xi)=\left(R_{t}\left(L_{t}(u, \xi)\right), Y_{t}\left(R_{t}\left(L_{t}(u, \xi)\right), \xi\right)\right)\right)
$$

is bounded in the appropriate norms. Let $L_{t}$ be given by (3.6) and $R_{t}$ be the flow of the reaction (3.4),

$$
\frac{d U}{d t}=f(U), \quad U(0)=u,
$$

where the reaction terms $f=\left(f_{1}, f_{2}, \ldots f_{N}\right)$ are given by

$$
f_{i}(U)=-\lambda U_{i}-q(U)
$$

where $\lambda=\operatorname{diag}\left(\lambda_{1}, \lambda_{2}, \ldots, \lambda_{N}\right)$ and $q=\left(q_{1}, q_{2}, \ldots, q_{N}\right)$ is given by

$$
q_{i}=\left(\sum_{j \neq i} r_{i j} U_{j}\right) U_{i} .
$$

Recall that the constants $\lambda_{i} \geq 0$ and $r_{i j} \geq 0$ for all $i, j=1, \ldots, N$. Define $\lambda_{\min }=$ $\min \left\{\lambda_{i}: i=1,2, \ldots, N\right\}$.

LEMma 4.7. Suppose $k$ is a nonnegative integer, $t \in[0, T)$ and $u \in \mathbb{H}_{k}^{N}$ such that $u_{i} \geq 0$ for $i=1,2, \ldots N$. Then

$$
\begin{aligned}
\left|R_{t}\left(L_{t}(u, \xi)\right)\right| & \leq\left(\sup |u|+C_{g} t\right) e^{-\lambda_{\min } t} \\
\left\|R_{t}\left(L_{t}(u, \xi)\right)\right\|_{k}^{2} & \leq\left(\|u\|_{k}^{2}+C_{g, k} t\right) e^{C_{f, k}(\sup |u|) t} .
\end{aligned}
$$

Proof. By Lemma 2.15,

$$
\begin{aligned}
\left|L_{t}(u, \xi)\right| & \leq\left|K_{t}(u)\right|+\int_{0}^{t}\left|K_{t-\tau}\left(g \circ \mathcal{T}_{\xi}\right)\right| d \tau \leq \sup |u|+\int_{0}^{t}\left|g \circ \mathcal{T}_{\xi}\right| d \tau \\
& \leq \sup |u|+C_{g} T
\end{aligned}
$$

and

$$
\begin{aligned}
\left\|L_{t}(u, \xi)\right\|_{k} & \leq\left\|K_{t}(u)\right\|_{k}+\int_{0}^{t}\left\|K_{t-\tau}\left(g \circ \mathcal{T}_{\xi}\right)\right\|_{k} d \tau \leq\|u\|_{k}+\int_{0}^{t}\left\|g \circ \mathcal{T}_{\xi}\right\|_{k} d \tau \\
& \leq\|u\|_{k}+C_{g, k} T
\end{aligned}
$$

Recall that the flow of (3.4) is defined by $\frac{d}{d t} R_{t}(u)=f\left(R_{t}(u)\right)$. Assume $u_{i}>0$ for $i=1,2, \ldots, N$. It follows from Lemma 4.6 that for $t>0, R_{t}\left(u_{i}\right)>0$ and $q_{i}\left(R_{t}(u)\right)>0$ for all $i$. Thus,

$$
\frac{d}{d t} R_{t}(u) \leq-\lambda R_{t}(u) \quad \text { and } \quad R_{t}(u) \leq u e^{-t \lambda}
$$


Recall that if $u_{i} \geq 0$ for all $i, L_{t}\left(u_{i}\right)>0$ for all $t>0$. Then by (4.22),

$$
\left|R_{t}\left(L_{t}(u)\right)\right| \leq\left|L_{t}(u)\right| e^{-\lambda_{\min } t} \leq\left(\sup |u|+C_{g} t\right) e^{-\lambda_{\min } t} .
$$

From Theorem 2.13 we have

$$
\begin{aligned}
\frac{1}{2} \frac{d}{d t}\left\|\partial^{\alpha} R_{t}(u)\right\|_{0}^{2} & =\left(\partial^{\alpha}\left(\frac{d}{d t} R_{t}(u)(t)\right), \partial^{\alpha} R_{t}(u)\right)=\left(\partial^{\alpha} f\left(R_{t}(u)\right), \partial^{\alpha} R_{t}(u)\right) \\
& \leq\left\|\partial^{\alpha} f\left(R_{t}(u)\right)\right\|_{0}\left\|\partial^{\alpha} R_{t}(u)\right\|_{0} .
\end{aligned}
$$

Summing over $|\alpha| \leq k$,

$$
\frac{1}{2} \frac{d}{d t}\left\|R_{t}(u)\right\|_{k}^{2} \leq C_{f, k}(\sup |u|)\left\|R_{t}(u)\right\|_{k}^{2} .
$$

The solution of the ordinary differential equation above is

$$
\left\|R_{t}(u)\right\|_{k}^{2} \leq\|u\|_{k}^{2} e^{C_{f, k}(\sup |u|) t}
$$

Equation (4.21) follows from substituting $L_{t}(u)$ for $u$ and applying (4.23).

Corollary 4.8. Let $j=1,2, \ldots, J$ and $C_{1}$ and $C_{2}$ be constants that are independent of $\delta$ for $\delta<1$. Under the assumptions of Lemma 4.7,

$$
\begin{aligned}
\left|V_{j}\right| & \leq \sup |u| e^{-\lambda_{\min } T}+C_{1}, \\
\left\|V_{j}\right\|_{k}^{2} & \leq\left(\|u\|_{k}+C_{2}\right) e^{C_{f, k}(\sup |u|) T} .
\end{aligned}
$$

Proof. Equations (4.25) and (4.26) follow from repeatedly applying (4.20) and (4.21), respectively.

LEMMA 4.9. If $Y_{t}(u, \xi)$ is a solution to (3.5), then

$$
\mathbb{E}\left[\sup _{0 \leq t \leq T}\left|Y_{t}(u, \xi)\right|^{2}\right] \leq C_{X}(\xi, T)
$$

Proof. Recall the stochastic differential equation for the velocity is

$$
d v_{t}=\left[h\left(U\left(x_{t}\right)\right)-\gamma v_{t}\right] d t+\sigma \sqrt{\gamma} d W_{t} .
$$

Using an integrating factor, $e^{\gamma t}$, the solution is

$$
v_{t}=e^{-\gamma t} \nu+\int_{0}^{t} e^{-\gamma(t-\tau)} h\left(U\left(x_{t}\right)\right) d \tau+\sigma \sqrt{\gamma} \int_{0}^{t} e^{\gamma(t-\tau)} d W_{\tau} .
$$

Therefore, by the Cauchy-Schwarz inequality, Doob martingale inequality and Itô Isometry,

$$
\begin{aligned}
\mathbb{E}\left[\sup _{0 \leq t \leq T}\left|v_{t}\right|^{2}\right] \leq & 3 e^{-2 \gamma T} \nu+3 \mathbb{E}\left[\sup _{0 \leq t \leq T}\left|\int_{0}^{t} e^{-\gamma(t-\tau)} h\left(U\left(x_{t}\right)\right) d \tau\right|^{2}\right] \\
& +3 \sigma^{2} \gamma \mathbb{E}\left[\sup _{0 \leq t \leq T}\left|\int_{0}^{t} e^{-\gamma(t-\tau)} d W_{\tau}\right|^{2}\right]
\end{aligned}
$$




$$
\begin{aligned}
\leq & 3 e^{-2 \gamma T} \nu+3 T \mathbb{E}\left[\sup _{0 \leq t \leq T} \int_{0}^{t} e^{-2 \gamma(t-\tau)}\left|h\left(U\left(x_{t}\right)\right)\right|^{2} d \tau\right] \\
& +12 \sigma^{2} \gamma \mathbb{E}\left[\sup _{0 \leq t \leq T}\left|\int_{0}^{t} e^{-\gamma(t-\tau)} d \tau\right|^{2}\right] \\
\leq & 3 e^{-2 \gamma T} \nu+\frac{3 T}{2 \gamma}\left(1-e^{-2 \gamma T}\right)+6 \sigma\left(1-e^{-2 \gamma T}\right) .
\end{aligned}
$$

The bound on $Y_{t}$ (4.27) immediately follows.

The following theorem gives the convergence rate of a general numerical scheme to approximate (2.19). The proof builds on an argument by Milstein in [9].

TheOREM 4.10. Let $p_{2} \geq \frac{1}{2}$ and $p_{1} \geq p_{2}+\frac{1}{2}$ and $k \geq 3$ be an integer. Assume that there exist constants $C_{1}, C_{2}>0$ such that

$$
\begin{gathered}
|\Phi(t, \xi, \delta)-\Psi(t, \xi, \delta)|_{k} \leq C_{1} \delta^{p_{1}}, \\
\left|\|\Phi(t, \xi, \delta)-\Psi(t, \xi, \delta) \mid\|_{k} \leq C_{2} \delta^{p_{2}} .\right.
\end{gathered}
$$

Then for $j=0,1 \ldots, J$, there exists a constant $C$ such that

$$
\left\|\Phi\left(t_{0}, \Phi_{0}, t_{j}\right)-\Psi\left(t_{0}, \Phi_{0}, t_{j}\right)\right\|_{k} \leq C \delta^{p_{2}-\frac{1}{2}} .
$$

Proof. In order to make use of Theorem 4.1 and conditions (4.28) and (4.29), we write

$$
\begin{aligned}
\Phi_{j+1}-\Psi_{j+1} & =\Phi\left(t_{j}, \Phi_{j}, \delta\right)-\Psi\left(t_{j}, \Psi_{j}, \delta\right) \\
& =\Phi\left(t_{j}, \Phi_{j}, \delta\right)-\Phi\left(t_{j}, \Psi_{j}, \delta\right)+T\left(t_{j}, \Psi_{j}, \delta\right) .
\end{aligned}
$$

Then

$$
\left\|\Phi_{j+1}-\Psi_{j+1}\right\|_{k}^{2}=A_{1}+2 A_{2}+A_{3}
$$

where

$$
\begin{aligned}
A_{1}= & \left\|\Phi\left(t_{j}, \Phi_{j}, \delta\right)-\Phi\left(t_{j}, \Psi_{j}, \delta\right)\right\|_{k}^{2}, \\
A_{2}= & \mathbb{E}\left[\left\|U\left(t_{j}, \Phi_{j}, \delta\right)-U\left(t_{j}, \Psi_{j}, \delta\right)\right\|_{k}\left\|T_{U}\left(t_{j}, \Psi_{j}, \delta\right)\right\|_{k}\right. \\
& \left.\quad+\left(X\left(t_{j}, \Phi_{j}, \delta\right)-X\left(t_{j}, \Psi_{j}, \delta\right)\right) T_{X}\left(t_{j}, \Psi_{j}, \delta\right)\right], \\
A_{3}= & \left\|T\left(t_{j}, \Psi_{j}, \delta\right)\right\|_{k}^{2} .
\end{aligned}
$$

By Theorem 4.1 and (4.29), we have

$$
A_{1} \leq\left\|\Phi_{j}-\Psi_{j}\right\|_{k}^{2}\left(1+C_{\Phi, k} \delta\right)=\left\|e_{j}\right\|_{k}^{2}\left(1+C_{\Phi, k} \delta\right)
$$

and

$$
A_{3} \leq C_{2} \delta^{2 p_{2}}
$$

respectively. Using Lemma 4.1, we write $A_{2} \leq B_{1}+B_{2}$ where

$$
B_{1}=\mathbb{E}\left[\left\|K_{\delta}\left(U_{j}-V_{j}\right)\right\|_{k}\left\|T_{U}\left(t_{j}, \Psi_{j}, \delta\right)\right\|_{k}+\left(X_{j}-Y_{j}\right) T_{X}\left(t_{j}, \Psi_{j}, \delta\right)\right]
$$


and

$$
B_{2}=\mathbb{E}\left[\left\|Z_{U}\right\|_{k}\left\|T_{U}\left(t_{j}, \Psi_{j}, \delta\right)\right\|_{k}+Z_{X} T_{X}\left(t_{j}, \Psi_{j}, \delta\right)\right] .
$$

Then by the Cauchy-Schwarz inequality and condition (4.28)

$$
\begin{aligned}
B_{1} & \leq \mathbb{E}\left[\mathbb{E}\left[\left\|U_{j}-V_{j}\right\|_{k}\left\|T_{U}\left(t_{j}, \Psi_{j}, \delta\right)\right\|_{k} \mid \mathcal{F}_{j}\right]\right]+\mathbb{E}\left[\mathbb{E}\left[\left(X_{j}-Y_{j}\right) \cdot T_{X}\left(t_{j}, \Psi_{j}, \delta\right) \mid \mathcal{F}_{j}\right]\right] \\
& \leq \mathbb{E}\left[\left\|U_{j}-V_{j}\right\|_{k} \mathbb{E}\left[\left\|T_{U}\left(t_{j}, \Psi_{j}, \delta\right)\right\|_{k} \mid \mathcal{F}_{j}\right]\right]+\mathbb{E}\left[\left(X_{j}-Y_{j}\right) \cdot \mathbb{E}\left[T_{X}\left(t_{j}, \Psi_{j}, \delta\right) \mid \mathcal{F}_{j}\right]\right] \\
& =\mathbb{E}\left[\left(\begin{array}{c}
\left\|U_{j}-V_{j}\right\|_{k} \\
X_{j}-Y_{j}
\end{array}\right) \cdot \mathbb{E}\left[\left(\begin{array}{c}
\left\|T_{U}\left(t_{j}, \Psi_{j}, \delta\right)\right\|_{k} \\
T_{X}\left(t_{j}, \Psi_{j}, \delta\right)
\end{array}\right) \mid \mathcal{F}_{j}\right]\right] \\
& \leq \mathbb{E}\left[\left|\left(\begin{array}{c}
\left\|U_{j}-V_{j}\right\|_{k} \\
X_{j}-Y_{j}
\end{array}\right)\right|^{2}\right]^{\frac{1}{2}} \mathbb{E}\left[\left|\mathbb{E}\left[\left(\begin{array}{c}
\left\|T_{U}\left(t_{j}, \Psi_{j}, \delta\right)\right\|_{k} \\
T_{X}\left(t_{j}, \Psi_{j}, \delta\right)
\end{array}\right) \mid \mathcal{F}_{j}\right]\right|^{2}\right]^{\frac{1}{2}} \\
& \leq\left\|e_{j}\right\|_{k}\left(\sqrt{2} C_{1} \delta^{p_{1}}\right) .
\end{aligned}
$$

Using the Cauchy-Schwarz inequality, (4.8) and (4.29) we estimate the second term,

$$
\begin{aligned}
B_{2} & =\mathbb{E}\left[\left(\begin{array}{c}
\left\|Z_{U}\right\|_{k} \\
Z_{X}
\end{array}\right) \cdot\left(\begin{array}{c}
\left\|T_{U}\left(t_{j}, \Psi_{j}, \delta\right)\right\|_{k} \\
T_{X}\left(t_{j}, \Psi_{j}, \delta\right)
\end{array}\right)\right] \\
& \leq \mathbb{E}\left[\left|\left(\begin{array}{c}
\left\|Z_{U}\right\|_{k} \\
Z_{X}
\end{array}\right)\right|^{2}\right]^{\frac{1}{2}} \mathbb{E}\left[\left|\left(\begin{array}{c}
\left\|T_{U}\left(t_{j}, \Psi_{j}, \delta\right)\right\|_{k} \\
T_{X}\left(t_{j}, \Psi_{j}, \delta\right)
\end{array}\right)\right|^{2}\right]^{\frac{1}{2}} \\
& =\|Z\|\left\|_{k}\right\| T\left(t_{j}, \Psi_{j}, \delta\right) \|_{k} \\
& \leq C_{\Phi, k}\left\|e_{j}\right\|_{k} \delta^{\frac{1}{2}}\left(C_{2} \delta^{p_{2}}\right) .
\end{aligned}
$$

Since $p_{1} \geq p_{2}+1, \delta^{p_{1}} \leq \delta^{p_{2}+\frac{1}{2}}$ for $\delta<1$. Thus,

$$
A_{2} \leq C\left\|e_{j}\right\|_{k} \delta^{p_{2}+\frac{1}{2}} \leq \frac{\delta\left\|e_{j}\right\|_{k}^{2}}{2}+\frac{C^{2} \delta^{2 p_{2}}}{2} .
$$

Putting the estimates for (4.31), (4.33) and (4.32) together,

$$
\left\|e_{j+1} \mid\right\|_{k}^{2} \leq\left\|e_{j}\right\|_{k}^{2}\left(1+C_{\Phi, k} \delta\right)+C \delta^{2 p_{2}} .
$$

The main result, (4.30), follows from Lemma 4.4 with $e_{0}=0$. $\square$

REMARK 4.11. The split scheme $\Psi$ from Chapter 3 satisfies Theorem (4.10) with $p_{1}=2$ and $p_{2}=\frac{3}{2}$ and is therefore a first order method.

4.4. Global Bounds. We now show that the solution to (2.19) is bounded in the appropriate norms for $t \in[0, T)$.

THEOREM 4.12. Let $\Phi(t)=\left(U(t), X_{t}\right)$ be a solution of the reaction-diffusionstochastic system (2.19) on $[0, T)$. Then for any nonnegative integer $k$ there exist constants $C_{U}$ and $C_{U, k}$ such that

$$
\begin{aligned}
\sup |U(t)| & \leq C_{U}(\sup |u|), \\
\|U(t)\|_{k} & \leq C_{U, k}\left(\sup |u|,\|u\|_{k}, T\right) .
\end{aligned}
$$

Proof. Using (4.18) and (4.19) we can rewrite the first equation in (2.19) as

$$
\frac{d U}{d t}=(\mathcal{D}-\lambda) U(t)+q(U(t))+g \circ \mathcal{T}_{X_{t}}, \quad U(0)=u
$$


where we assume that $u \geq 0$. Define $\mathcal{K}_{t}=e^{-t \lambda} K_{t}$. Then

$$
\begin{aligned}
\frac{d}{d t} \mathcal{K}_{t}(u) & =e^{-t \lambda}\left(\frac{d}{d t} K_{t}(u)-\lambda K_{t}(u)\right) \\
& =e^{-t \lambda}\left(\mathcal{D} K_{t}(u)-\lambda K_{t}(u)\right) \\
& =\mathcal{K}_{t}[(\mathcal{D}-\lambda) u] .
\end{aligned}
$$

Therefore,

$$
U(t)=\mathcal{K}_{t}(u)+\int_{0}^{t} \mathcal{K}_{t-\tau}\left(q(U)+g \circ \mathcal{T}_{X_{\tau}}\right) d \tau
$$

Clearly $q_{i}(U)<0$ for all $i$ and thus

$$
U(t) \leq \mathcal{K}_{t}(u)+\int_{0}^{t} \mathcal{K}_{t-\tau}\left(g \circ \mathcal{T}_{X_{\tau}}\right) d \tau .
$$

Then (4.35) follows from the fact that

$$
|U(t)| \leq\left|\mathcal{K}_{t} u\right|+\int_{0}^{t} e^{-(t-\tau) \lambda}\left|K_{t}\left(g \circ \mathcal{T}_{X_{\tau}}\right)\right| d \tau \leq|u|+\frac{1}{\lambda} C_{g} .
$$

Let $u, v \in \mathbb{H}_{k}^{N}$ and $|\alpha| \leq k$ for a nonnegative integer $k$. Then,

$$
\begin{aligned}
\left(\partial^{\alpha} u, \partial^{\alpha} v\right) & =\sum_{i=1}^{N}\left(\partial^{\alpha} u_{i}, \partial^{\alpha} v_{i}\right) \\
& \leq \frac{1}{2} \sum_{i=1}^{N}\left(\left\|\partial^{\alpha} u_{i}\right\|_{k}^{2}+\left\|\partial^{\alpha} v_{i}\right\|_{k}^{2}\right) \\
& =\frac{1}{2}\left(\left\|\partial^{\alpha} u\right\|_{k}^{2}+\left\|\partial^{\alpha} v\right\|_{k}^{2}\right) .
\end{aligned}
$$

Thus, using Theorem 2.13 and Lemma 2.15,

$$
\begin{aligned}
\frac{1}{2} \frac{d}{d t}\left\|\partial^{\alpha} U(t)\right\|_{0}^{2} & =\left(\partial^{\alpha} \dot{U}(t), \partial^{\alpha} U(t)\right) \\
& =\left(\mathcal{D} \partial^{\alpha} U(t), \partial^{\alpha} U(t)\right)+\left(\partial^{\alpha} f(U(t)), \partial^{\alpha} U(t)\right)+\left(\partial^{\alpha} g, \partial^{\alpha} U(t)\right) \\
& \leq\left\|\partial^{\alpha} f(U(t))\right\|_{0}\left\|\partial^{\alpha} U(t)\right\|_{0}+\frac{1}{2}\left(\left\|\partial^{\alpha} g\right\|_{0}^{2}+\left\|\partial^{\alpha} U(t)\right\|_{0}^{2}\right)
\end{aligned}
$$

Summing over $|\alpha| \leq k$,

$$
\frac{1}{2} \frac{d}{d t}\|U(t)\|_{k}^{2} \leq C_{f, k}\left(C_{U}(\sup |u|)\right)\|U(t)\|_{k}^{2}+\frac{1}{2} C_{g, k}^{2} .
$$

The estimate (4.36) follows from the fact that the solution of the ordinary differential equation above is

$$
\|U(t)\|_{k}^{2} \leq\left(\|u\|_{k}^{2}+\frac{C_{g, k}^{2}}{C_{f, k}\left(C_{U}(\sup |u|)\right)}\right) e^{C_{f, k}\left(C_{U}(\sup |u|)\right) t}-\frac{C_{g, k}^{2}}{C_{f, k}\left(C_{U}(\sup |u|)\right)} .
$$

$\square$ 
4.5. Long Time Existence. We now show that the solution to the reactiondiffusion-stochastic system exists for all time.

THEOREM 4.13. The solution of (2.19) exists for all $t \geq 0$.

Proof. Suppose that $[0, T)$ is the largest interval on which $\Phi(t)$ is a solution of the reaction-diffusion-stochastic system (2.19). If $\lim _{t \uparrow T} \Phi(t)$ exists in $\mathcal{C}_{k}^{N}$, then there exists a solution on the interval $[0, T+\delta)$ for some $\delta>0$ that depends on the appropriate bounds on $\Phi(t)$. But this contradicts the assumption that $[0, T)$ is the largest interval of existence and therefore, the solution of (2.19) exists for all time.

In order to show that $\lim _{t \uparrow T} \Phi(t)$ exists, we obtain the Hölder estimate

$$
\|\Phi(t+\delta)-\Phi(t)\|_{k} \leq C \delta^{\frac{1}{2}}
$$

for some constant $C$. Suppose $U(0)=u$. Then by Theorem 2.13, Lemma 2.15 and $(4.35)$,

$$
\begin{aligned}
\|U(t+\delta)-U(t)\|_{k} & \leq\left\|\left(K_{\delta}-1\right) K_{t}(u)\right\|_{k}+\int_{t}^{t+\delta}\|f(U(\tau))\|_{k}+\left\|g \circ \mathcal{T}_{X_{\tau}}\right\|_{k} d \tau \\
& \leq \delta^{\frac{1}{2}}\|u\|_{k+1}+\left[C_{f, k}\left(C_{U}(\sup |u|), C_{U, k}\left(\sup |u|,\|u\|_{k}, T\right)+C_{g, k}\right] \delta\right.
\end{aligned}
$$

By (2.33), (4.36) and (4.27), we have

$$
\begin{aligned}
\mathbb{E}\left[\left|X_{t+\delta}-X_{t}\right|^{2}\right] & \leq \mathbb{E}\left[2\left|\int_{t}^{t+\delta} a\left(U\left(X_{\tau}\right), X_{\tau}\right) d \tau\right|^{2}+2\left|\int_{t}^{t+\delta} b\left(X_{\tau}\right) d W_{\tau}\right|^{2}\right] \\
& \leq \mathbb{E}\left[2 \delta\left|\int_{t}^{t+\delta} a\left(U\left(X_{\tau}\right), X_{\tau}\right) d \tau\right|^{2}+2 \int_{t}^{t+\delta}\left|b\left(X_{\tau}\right)\right| d \tau\right]^{2} \\
& \leq 2 \delta^{2} C_{a}(\xi, T)+2 \sigma^{2} \gamma \delta .
\end{aligned}
$$

Therefore, (4.40) holds with $C=C\left(\sup |u|,\|u\|_{k}, \xi, T\right)$.

5. Conclusions. The use of a first order splitting for the reaction-diffusionstochastic system allows us to make use of known first order numerical methods for the diffusion, reaction and stochastic differential equations. In particular, the diffusion can be discretized spatially using finite elements. From [1], we know that the resulting error is $\mathcal{O}\left(h^{2}\right)$ where $h$ is the grid spacing. The resulting problem can be discretized in time using a first order backward Euler scheme as described in [12]. From [3] we know that the backward Euler scheme preserves positivity. The reaction can be approximated by a semi-implicit scheme that preserves positivity. General numerical methods for stochastic differential equations are outlined in [6]. In our case the cell motion (1.2), is a Langevin process that can be simulated exactly using ideas from [7]. By combining the numerical methods outlined above we obtain a numerical simulation that is first order in time and preserves the positivity of the soluble factors.

Figure 5 shows a simulation of the immune response to three sources of MCP1 which is known to attract immune cells called macrophages. A comparison of 1(a) and 1(b) shows that the macrophages are attracted to the three sources of MCP1 in the simulation. The macrophages also secrete the chemokines TNF and sTNFr which is shown in $1(\mathrm{c})$ and $1(\mathrm{~d})$. The diffusion of all three soluble factors is also apparent in Figure 5. 


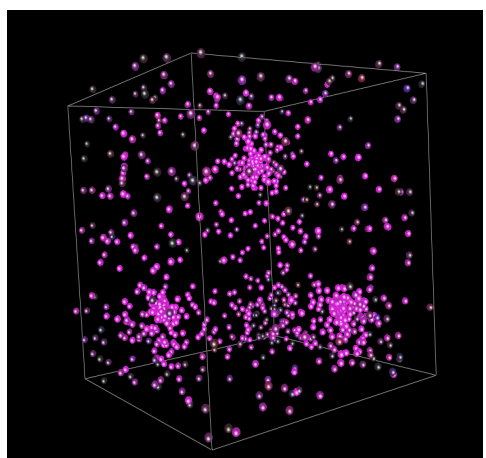

(a) Macrophages responding to $\mathrm{MCP} 1$

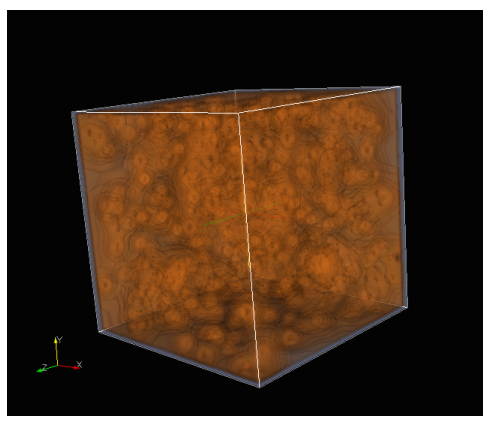

(c) Concentration of TNF

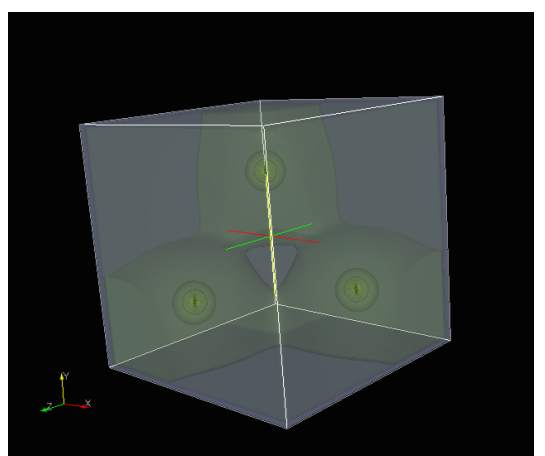

(b) Concentration of MCP1

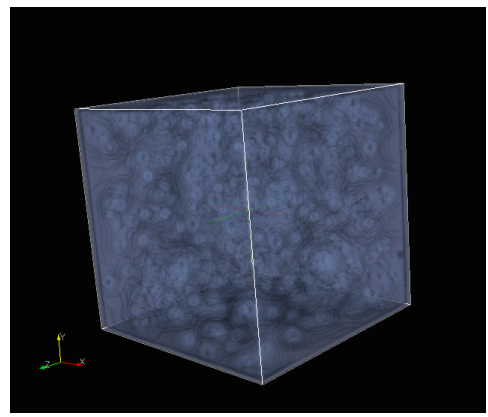

(d) Concentration of sTNFr

FIG. 5.1. Simulation of an immune response to three sources of MCP1.

\section{REFERENCES}

[1] Susanne C. Brenner and L. Ridgway Scott, The mathematical theory of finite element methods, Springer-Verlag, New York, second ed., 2002.

[2] Gerald B. Folland, Introduction to Differential Equations, Princeton University Press, Princeton, NJ, second ed., 1995.

[3] Willem Hundsdorfer And Jan Verwer, Numerical solution of time-dependent advectiondiffusion-reaction equations, Springer-Verlag, Berlin, 2003.

[4] Thomas B. Kepler, Microsimulation of inducible reorganization in immunity, in Complex Systems Science in BioMedicine, Thomas S. Deisboeck and J. Yasha Kresh, eds., International Topics in Biomedical Engineering, Springer, New York, 2006, pp. 437-450.

[5] Thomas B Kepler and Cliburn Chan, Spatiotemporal programming of a simple inflammatory process., Immunol Rev, 216 (2007), pp. 153-163.

[6] P.E. Kloeden and E. Platen, Numerical Solution of Stochastic Differential Equations, Springer, Berlin, 1999.

[7] Don S. Lemons, An introduction to stochastic processes in physics, Johns Hopkins University Press, Baltimore, MD, 2002. Translated by Anthony Gythiel.

[8] Xuerong MaO, Stochastic differential equations and their applications, Horwood Publishing Limited, Chichester, 1997.

[9] G. N. MiLstein, Numerical integration of stochastic differential equations, Kluwer Academic Publishers Group, Dordrecht, 1995. Translated and revised from the 1988 Russian original.

[10] Bernt ØKsendal, Stochastic differential equations, Universitext, Springer-Verlag, Berlin, sixth ed., 2003. An introduction with applications.

[11] Michael E. Taylor, Partial differential equations. III, Springer-Verlag, New York, 1997. Nonlinear equations, Corrected reprint of the 1996 original.

[12] Vidar Thomée, Galerkin finite element methods for parabolic problems, Springer-Verlag, Berlin, second ed., 2006. 